TU PREPRINT

TU-E18-06-0801

tupaper11.tex

\title{
Covariant Helicity-Coupling Amplitudes: A New Formulation
}

\author{
Suh-Urk Chung * and Jan Michael Friedrich \\ Physik Department E18, Technische Universität München, Germany
}

October 25, 2018

\begin{abstract}
We have worked out covariant amplitudes for any two-body decay of a resonance with an arbitrary non-zero mass, which involves arbitrary integer spins in the initial and the final states. One key new ingredient for this work is the application of the total intrinsic spin operator $\vec{S}$ which is given directly in terms of the generators of the Poincaré group.

Using the results of this study, we show how to explore the Lorentz factors which appear naturally, if the momentum-space wave functions are used to form the covariant decay amplitudes. We have devised a method of constructing our covariant decay amplitudes, such that they lead to the Zemach amplitudes when the Lorentz factors are set one.
\end{abstract}

* Mercator Visiting Professor for 2003-2005 and 2007-2009

Permanent Address: Physics Department, Brookhaven National Laboratory,

Upton, NY 11973, USA 


\section{Introduction}

When one attempts to write down the covariant amplitudes for two-body decay processes involving arbitrary spin, one discovers invariably that the Lorentz factors $\gamma$ (defined as the energy for each of the decay products divided by its invariant mass) appear in the amplitudes. This is an endemic feature of any phenomenological amplitude one writes down in fourmomentum space. The purpose of this paper is to systematically investigate functional dependence of such Lorentz factors.

In previous publications and notes by one of the authors[1] [2], a few of the more relevant examples of this problem have been worked out, but it was all done under the assumption that the total intrinsic spin can be derived from 'fictitious' wave functions of the decay products, which are deemed to be at rest in the parent rest frame. We show in this paper how this problem could be properly handled in the general relativistic frame work - the key ideas involved are succinctly summarized in Section 2. Consider the canonical state $|\vec{p}, j m\rangle$ of a single particle. A rotation operator which acts on the state modifies both $\vec{p}$ and $|j m\rangle$, the prescription of which is very well known and it involves an introduction of angular momentum operators $\vec{J}$ in the expression for the rotation operators. There exists, however, a new subgroup of rotations of $S U(2)$ which leaves $\vec{p}$ invariant, affecting only the $|j m\rangle$. This is accomplished by replacing $\vec{J}$ in the rotation operator with $\vec{S}$, but now the $\vec{S}$ becomes a complicated function of all the generators of the Poincaré group, i.e. $\vec{J}$ (angular momentum operator), $\vec{K}$ (the boost operator) and $\vec{P}$ (the translation operator). It has been shown that the rotation operator involving $\vec{L}=\vec{J}-\vec{S}$ instead of $\vec{S}$ affects $\vec{p}$ while leaving $|j m\rangle$ invariant[4][5]. The operators $\vec{S}$ and $\vec{L}$ provide fully relativistic prescription for the familiar $L S$ coupling scheme for two-particle final states. The application of this concept to the problem of exploring the functional dependence of the Lorentz factors leads to the generators of the Lorentz group in four-momentum space, in which the operators $\vec{P}$ is replaced by their eigenvalues $\vec{p}$.

In Sections 3 and 4, we start from general wave functions at rest of arbitrary integer spin and a description of the kinematics of the two-body decay; much of these results have been worked out by one of us in a previous publication[2], but they are reproduced here for ease of reference. We cover in Section 5 the general wave functions in a given total intrinsic spin. In Section 6, we cover the rules for constructing covariant helicity-coupling amplitudes - this 
constitutes the heart of what is new in this paper. A number of illustrative examples are given in Sections 7, 8 and 9, for the decay amplitudes in which decay products have spin 0, 1 or 2. We have worked out in Section 10 an example of a sequence of decay chains: a parent state of spin $J$ decays into two intermediate states of spins $s$ and $\sigma$, each of which decays into $2 \pi$ and $3 \pi$, respectively. We have given in some detail a possible set of angles defined in two helicity coordinate systems, the rest frames of $s$ and $\sigma$, which are needed to describe the secondary decays. Section 11 is reserved for conclusions and discussions.

The Appendix A deals with the generators of the Lorentz group, starting with the general rank-2 angular momentum tensor in four-momentum space. In the Appendix B, we have worked out a derivation of the spherical harmonics from the tensor wave functions $\phi(J \mathrm{~m})$ given in Section 4. The Appendix $\mathrm{C}$ deals with certain additional decay amplitudes not included in the main text, to show why and how they have been excluded in the construction of covariant decay amplitudes in our paper; the excluded decay amplitudes violate the $r^{\ell}$ rule, i.e. an orbital angular momentum $\ell$ must induce an $r^{\ell}$ dependence in the amplitude, where $r$ is the relative momentum of the daughter states in the parent rest frame.

\section{Relativistic Two-Body Systems}

Consider a two-body decay $J \rightarrow s+\sigma$. The canonical states for the decay products are denoted $\left|\vec{q}, s m_{s}\right\rangle$ and $\left|\vec{k}, \sigma m_{\sigma}\right\rangle$, where $\vec{q}$ and $\vec{k}$ stand for the momenta for the daughter particles. So $\vec{q}=-\vec{k}$ in the $J$ rest frame $(J \mathrm{RF})$.

In this paper we use the word 'relativistic' to mean that the magnitude of the momentum $q$ or $k$ can take on arbitrary values and, therefore, that the Lorentz factors $\gamma_{s}>1$ or $\gamma_{\sigma}>1$ can be arbitrarily large. Conversely, the word 'non-relativistic' means that a statement is true only in the limit $q \approx 0$ or $k \approx 0$ and $\gamma_{s} \approx 1$ or $\gamma_{\sigma} \approx 1$.

The central idea used in this paper can be summarized as follows. Consider a relativistic canonical state for $s$, i.e. $\left|\vec{q}, s m_{s}\right\rangle$. We know how it transforms under an arbitrary rotation $R(\alpha, \beta, \gamma)$

$$
R(\alpha, \beta, \gamma)\left|q_{i}, s m_{s}\right\rangle=\sum_{m_{s}^{\prime} k}\left|R_{i k} q_{k}, s m_{s}^{\prime}\right\rangle D_{m_{s}^{\prime} m_{s}}^{s}(\alpha, \beta, \gamma), \quad i, k=1,2,3
$$


where $R$ on the left-hand side is the usual rotation operator given by

$$
R(\alpha, \beta, \gamma)=\exp \left[-i \alpha J_{z}\right] \exp \left[-i \beta J_{y}\right] \exp \left[-i \gamma J_{z}\right]
$$

and $R_{i k}$ on the right-hand side is the corresponding $3 \times 3$ matrix. It has been shown [4] [5] that the angular momentum operator $\vec{J}$ can be broken up into two components

$$
\vec{J}=\vec{S}+\vec{L}
$$

where, with $w$ denoting the mass of a particle,

$$
w \vec{S}=P^{0} \vec{J}-\vec{P} \times \vec{K}-\frac{1}{P^{0}+w} \vec{P}(\vec{P} \cdot \vec{J})
$$

$\vec{J}, \vec{K}$ and $\left(P^{0}, \vec{P}\right)$ are the generators of the Poincaré group. Here $\vec{L}$ is a derived quantity, defined by $\vec{L}=\vec{J}-\vec{S}$. The operators $\vec{S}$ and $\vec{L}$ satisfy the standard Lie algebra of the rotation group and they commute

$$
\begin{aligned}
& {\left[S_{i}, S_{j}\right]=i \epsilon_{i j k} S_{k}, \quad\left[L_{i}, L_{j}\right]=i \epsilon_{i j k} L_{k}, \quad\left[S_{i}, L_{j}\right]=0, \quad i, j, k=1,2,3} \\
& {\left[P^{0}, L_{i}\right]=0, \quad\left[P_{i}, L_{j}\right]=i \epsilon_{i j k} P_{k}, \quad \vec{P} \cdot \vec{L}=0}
\end{aligned}
$$

It was shown[4] that

$$
\begin{aligned}
& R^{S}(\alpha, \beta, \gamma)\left|q_{i}, s m_{s}\right\rangle=\sum_{m_{s}^{\prime}}\left|q_{i}, s m_{s}^{\prime}\right\rangle D_{m_{s}^{\prime} m_{s}}^{s}(\alpha, \beta, \gamma) \\
& R^{L}(\alpha, \beta, \gamma)\left|q_{i}, s m_{s}\right\rangle=\sum_{k}\left|R_{i k} q_{k}, s m_{s}\right\rangle
\end{aligned}
$$

where

$$
\begin{aligned}
& R^{S}(\alpha, \beta, \gamma)=\exp \left[-i \alpha S_{z}\right] \exp \left[-i \beta S_{y}\right] \exp \left[-i \gamma S_{z}\right] \\
& R^{L}(\alpha, \beta, \gamma)=\exp \left[-i \alpha L_{z}\right] \exp \left[-i \beta L_{y}\right] \exp \left[-i \gamma L_{z}\right]
\end{aligned}
$$

The equations (2.6a) and (2.6b) show that the operators $\vec{S}$ and $\vec{L}$ induce separate rotations on $\vec{q}$ and $\left|s m_{s}\right\rangle$, which are normally transformed simultaneously in a conventional rotation [see (2.1) ]. Thus $R^{S}(\alpha, \beta, \gamma)$ acts on the relativistic state $\left|q_{i}, s m_{s}\right\rangle$ as if it were a rest state, affecting only the spin components, whereas $R^{L}(\alpha, \beta, \gamma)$ acts on the relativistic state $\left|q_{i}, s m_{s}\right\rangle$ as if it were a spinless state, leaving the spin components invariant.

We are now ready to apply $R^{S}(\alpha, \beta, \gamma)$ and $R^{L}(\alpha, \beta, \gamma)$ to relativistic two-body systems. Consider a system consisting of two particles with momenta $\vec{q}$ and $\vec{k}$ with their spin states given by $\left|s m_{s}\right\rangle$ and $\left|\sigma m_{\sigma}\right\rangle$. Define

$$
\vec{S}=\vec{S}_{s}+\vec{S}_{\sigma} \quad \text { and } \quad \vec{L}=\vec{L}_{s}+\vec{L}_{\sigma}
$$


We define a two-body system in its rest frame (i.e. $\vec{q}+\vec{k}=0$ and $\vec{r}=\vec{q}-\vec{k}$ ) by

$$
|\vec{r} S m\rangle=\sum_{m_{s} m_{\sigma}}\left(s m_{s} \sigma m_{\sigma} \mid S m\right)\left|\vec{q}, s m_{s}\right\rangle\left|\vec{k}, \sigma m_{\sigma}\right\rangle
$$

We use the notation $\left(s_{1} m_{1} s_{2} m_{2} \mid S m_{s}\right)$ to stand for the standard Clebsch-Gordan coefficients [6]. Under a rotation $R^{S}$, the above state transforms as

$$
\begin{aligned}
& R^{S}(\phi, \theta, 0)\left|r_{i}, S m\right\rangle=\sum_{m^{\prime}}\left|r_{i}, S m^{\prime}\right\rangle D_{m^{\prime} m}^{S}(\phi, \theta, 0), \quad i=1,2,3 \\
& R^{L}(\phi, \theta, 0)\left|r_{i}, S m\right\rangle=\left|R_{i k} r_{k}, S m\right\rangle, \quad i, k=1,2,3
\end{aligned}
$$

where $\Omega=(\theta, \phi)$ describes the direction of $\vec{r}$ (as well as $\vec{q}$ and $\vec{k}$ ) in the $J$ RF. This shows the state $|\vec{r}, S m\rangle$ acts like a rest state under $R^{S}(\phi, \theta, 0)$ in the sense that $\vec{r}$ remains invariant. So we have established the concept of a total intrinsic spin $S$ for a relativistic two-body system. The operator $R^{L}(\phi, \theta, 0)$ acts only on the $\vec{r}$ as if the two final-state particles involved were spinless.

It is clear that this leads naturally to the concept of orbital angular momentum for the relativistic two-body system

$$
\left|\ell m_{\ell} S m\right\rangle \propto \int \mathrm{d} \Omega Y_{m_{\ell}}^{\ell}(\Omega)|\vec{r}, S m\rangle=\sqrt{\frac{2 \ell+1}{4 \pi}} \int \mathrm{d} \Omega D_{m 0}^{\ell *}(\phi, \theta, 0)|\vec{r}, S m\rangle
$$

The analogue of (2.10) for this ket state is

$$
\begin{aligned}
& R^{S}(\phi, \theta, 0)\left|\ell m_{\ell} S m\right\rangle=\sum_{m^{\prime}}\left|\ell m_{\ell} S m^{\prime}\right\rangle D_{m^{\prime} m}^{S}(\phi, \theta, 0) \\
& R^{L}(\phi, \theta, 0)\left|\ell m_{\ell} S m\right\rangle=\sum_{m_{\ell}^{\prime}}\left|\ell m_{\ell}^{\prime} S m\right\rangle D_{m_{\ell}^{\prime} m_{\ell}}^{\ell}(\phi, \theta, 0)
\end{aligned}
$$

The formula $(2.12 b)$ can be shown by substituting (2.9) into (2.11) and carrying out the actions of $R^{L}$ on the vectors $\vec{q}=\vec{r} / 2$ and $\vec{k}=-\vec{r} / 2$ as given in (2.10b). From (2.2), (2.3) and (2.5), we find that $R=R^{S} R^{L}=R^{L} R^{S}$ and so, in the $J R F$,

$$
R(\phi, \theta, 0)\left|\ell m_{\ell} S m\right\rangle=\sum_{m^{\prime} m_{\ell}^{\prime}}\left|\ell m_{\ell}^{\prime} S m^{\prime}\right\rangle D_{m_{\ell}^{\prime} m_{\ell}}^{\ell}(\phi, \theta, 0) D_{m^{\prime} m}^{S}(\phi, \theta, 0)
$$

which shows that the state $\left|\ell m_{\ell} S m\right\rangle$ is a product of two states $\left|\ell m_{\ell}\right\rangle$ and $|S m\rangle$ at rest in the JRF. We can thus derive the standard expansion of the relativistic two-body system in the $\ell S$-coupling scheme

$$
|J M \ell S\rangle=\sum_{m_{\ell} m}\left(\ell m_{\ell} S m \mid J M\right)\left|\ell m_{\ell} S m\right\rangle
$$


or

$$
\begin{aligned}
|J M \ell S\rangle \propto \sum_{m_{\ell} m}\left(\ell m_{\ell} S m \mid J M\right) \sum_{m_{s} m_{\sigma}}\left(s m_{s} \sigma m_{\sigma} \mid S m\right) \\
\times \int \mathrm{d} \Omega Y_{m_{\ell}}^{\ell}(\Omega)\left|\vec{q}, s m_{s}\right\rangle\left|\vec{k}, \sigma m_{\sigma}\right\rangle
\end{aligned}
$$

where $\vec{q}=\vec{r} / 2$ and $\vec{k}=-\vec{r} / 2$ in the $J$ RF. We obtain

$$
R(\phi, \theta, 0)|J M \ell S\rangle=\sum_{M^{\prime}}\left|J M^{\prime} \ell S\right\rangle D_{M^{\prime} M}^{J}(\phi, \theta, 0)
$$

This is the standard result, showing that $J, S$ and $\ell$ are rotational invariants.

We may summarize the results of this section as follows: There exist two ket states, $|S m\rangle$ and $\left|\ell m_{\ell}\right\rangle$, in the decay process $J \rightarrow s+\sigma$. They act like the states at rest in the $J R F$, as shown in $(2.12 a)$ and $(\underline{2.12 b})$. So there exist three at-rest states in the problem; $|J M\rangle$ in the initial system and $\left|\ell m_{\ell}\right\rangle$ in the final system, at rest in the $J R F$. The third state $|S m\rangle$ acts as if it is again at rest in the $J R F$, but it is composed of two states $\left|\vec{r} / 2, s m_{s}\right\rangle$ and $\left|-\vec{r} / 2, \sigma m_{\sigma}\right\rangle$ "in motion" in the JRF. We emphasize here that this is true even when the parent mass becomes arbitraily large; the state in a total intrinsic spin $S$, as given by (2.9), acts like a state at rest in the JRF. We shall exploit this in Section 6 to derive our covariant decay amplitudes in the momentum space.

\section{Two-Body Decay $J \rightarrow s+\sigma$}

Consider a two-body decay $J \rightarrow s+\sigma$, where we use the notations very similar to those introduced in a previous paper[1]. The daughter 1 with spin $s$ is described by a wave function $\omega(\lambda)$ and the daughter 2 by $\varepsilon(-\nu) \mathrm{a}$; see Table I for all other relevant quantities.

a The momentum of the daughter $\sigma$ is pointed toward the negative $z$-axis, as shown by $k^{\alpha}$ in (3.1). By definition, its helicity $\nu$ is measured along $\vec{k}$; as a consequence, its spin projection along the positive $z$-axis is $-\nu$. 
Table I. Two-body decay: $J \rightarrow s+\sigma$

\begin{tabular}{|l|c|c|c|}
\hline & Parent & Daughter 1 & Daughter 2 \\
\hline Spin & $J$ & $s$ & $\sigma$ \\
Parity & $\eta_{J}$ & $\eta_{s}$ & $\eta_{\sigma}$ \\
Helicity & & $\lambda$ & $\nu$ \\
Momentum & $\boldsymbol{p}$ & $\boldsymbol{q}$ & $\boldsymbol{k}$ \\
Energy & $p_{0}$ & $q_{0}$ & $k_{0}$ \\
Mass & $w$ & $w_{s}$ & $w_{\sigma}$ \\
Energy/Mass & & $\gamma_{s}$ & $\gamma_{\sigma}$ \\
Velocity & & $\beta_{s}$ & $\beta_{\sigma}$ \\
Wave function & $\phi^{*}(\lambda-\nu)$ & $\omega(\lambda)$ & $\varepsilon(-\nu)$ \\
\hline
\end{tabular}

The polarization four-vectors or wave functions appropriate for the particles $J=1$, ' $s=1$ ' and ' $\sigma=1$ ' are well known. Along with the relevant momenta,

$$
\begin{aligned}
& p^{\alpha}=(\quad w ; 0,0, \quad 0 \quad) \\
& q^{\alpha}=\left(\quad q_{0} ; 0, \quad 0, \quad q\right) \\
& =\left(\begin{array}{llll}
\gamma_{s} w_{s} ; & 0, & 0, & \gamma_{s} \beta_{s} w_{s}
\end{array}\right) \\
& k^{\alpha}=\left(k_{0} ; 0,0, \quad 0, \quad-q\right) \\
& =\left(\gamma_{\sigma} w_{\sigma} ; 0,0, \quad-\gamma_{\sigma} \beta_{\sigma} w_{\sigma}\right) \\
& r^{\alpha}=\left(\begin{array}{llll}
q_{0}-k_{0} ; 0, & 0, & 2 q
\end{array}\right)
\end{aligned}
$$

where $w=q_{0}+k_{0}, q_{0}=\sqrt{w_{s}^{2}+q^{2}}, k_{0}=\sqrt{w_{\sigma}^{2}+q^{2}}$ and $r=q-k$, the wave functions in the $J$ RF are given by

$$
\begin{aligned}
& \phi^{\alpha}( \pm)=\mp \frac{1}{\sqrt{2}}(\quad 0 ; \quad 1, \quad \pm i, \quad 0 \quad) \\
& \phi^{\alpha}(0)=(0 ; \quad 0, \quad 0, \quad 1 \quad) \\
& \omega^{\alpha}( \pm)=\mp \frac{1}{\sqrt{2}} \quad(\quad 0 ; \quad 1, \quad \pm i, \quad 0 \quad) \\
& \omega^{\alpha}(0)=\left(\gamma_{s} \beta_{s} ; 0, \quad 0, \gamma_{s}\right) \\
& \varepsilon^{\alpha}( \pm)=\mp \frac{1}{\sqrt{2}}(\quad 0 ; \quad 1, \quad \pm i, \quad 0 \quad) \\
& \varepsilon^{\alpha}(0)=\left(-\gamma_{\sigma} \beta_{\sigma} ; 0, \quad 0, \gamma_{\sigma}\right)
\end{aligned}
$$

Note that

$$
p_{\alpha} \phi^{\alpha}(\lambda)=q_{\alpha} \omega^{\alpha}(\lambda)=k_{\alpha} \varepsilon^{\alpha}(\lambda)=0
$$


for any $\lambda$.

These polarization four-vectors satisfy

$$
\begin{aligned}
p_{\alpha} \phi^{\alpha}(m) & =0 \\
\phi_{\alpha}^{*}(m) \phi^{\alpha}\left(m^{\prime}\right) & =-\delta_{m m^{\prime}} \\
\sum_{m} \phi_{\alpha}(m) \phi_{\beta}^{*}(m) & =\tilde{g}_{\alpha \beta}(w)
\end{aligned}
$$

where

$$
\tilde{g}_{\alpha \beta}(w)=-g_{\alpha \beta}+\frac{p_{\alpha} p_{\beta}}{w^{2}}
$$

The last equation of (3.3) is the usual projection operator for spin-1 states[1]. Note that, in the $J$ RF, $\tilde{g}(w)$ has a zero time-component and +1 for the space-components, i.e.

$$
\tilde{g}_{\alpha \beta}(w)=\tilde{g}^{\alpha \beta}(w)=\left(\begin{array}{cccc}
0 & 0 & 0 & 0 \\
0 & 1 & 0 & 0 \\
0 & 0 & 1 & 0 \\
0 & 0 & 0 & 1
\end{array}\right)
$$

$\omega$ and $\varepsilon$ satisfy similar conditions, but with their own $\tilde{g}$ 's, i.e., $\tilde{g}\left(w_{s}\right)$ and $\tilde{g}\left(w_{\sigma}\right)$

$$
\begin{aligned}
& \tilde{g}_{\alpha \beta}\left(w_{s}\right)=-g_{\alpha \beta}+\frac{q_{\alpha} q_{\beta}}{w_{s}^{2}} \\
& \tilde{g}_{\alpha \beta}\left(w_{\sigma}\right)=-g_{\alpha \beta}+\frac{k_{\alpha} k_{\beta}}{w_{\sigma}^{2}}
\end{aligned}
$$

so that

$$
\begin{aligned}
q_{\alpha} \omega^{\alpha}(m) & =0 \\
\omega_{\alpha}^{*}(m) \omega^{\alpha}\left(m^{\prime}\right) & =-\delta_{m m^{\prime}} \\
\sum_{m} \omega_{\alpha}(m) \omega_{\beta}^{*}(m) & =\tilde{g}_{\alpha \beta}\left(w_{s}\right)
\end{aligned}
$$

and

$$
\begin{aligned}
k_{\alpha} \varepsilon^{\alpha}(m) & =0 \\
\varepsilon_{\alpha}^{*}(m) \varepsilon^{\alpha}\left(m^{\prime}\right) & =-\delta_{m m^{\prime}} \\
\sum_{m} \varepsilon_{\alpha}(m) \varepsilon_{\beta}^{*}(m) & =\tilde{g}_{\alpha \beta}\left(w_{\sigma}\right)
\end{aligned}
$$




\section{Spin- $J$ Wave Functions}

The general spin- $J$ wave function can be written[1], with $m \geq 0$

$$
\begin{gathered}
\phi_{\delta_{1} \cdots \delta_{J}}(J m)=\left[a^{J}(m)\right]^{\frac{1}{2}} \sum_{m_{0}} 2^{\left(m_{0}-\kappa\right) / 2} \sum_{P} \underbrace{\phi_{\alpha_{1}}(+) \cdots}_{m_{+}} \underbrace{\phi_{\beta_{1}}(0) \cdots}_{m_{0}} \underbrace{\phi_{\gamma_{1}}(-) \cdots}_{m_{-}} \\
\text {with } a^{J}(m)=\frac{2^{\kappa}(J+m) !(J-m) !}{(2 J) !}
\end{gathered}
$$

The indices $\left\{\delta_{1} \cdots \delta_{J}\right\}$ are grouped into $\left\{\alpha_{i}\right\}$ with $\left(i=1, m_{+}\right),\left\{\beta_{i}\right\}$ with $\left(i=1, m_{0}\right)$ and $\left\{\gamma_{i}\right\}$ with $\left(i=1, m_{-}\right)$, where $m_{ \pm}$stands for the number of $\phi( \pm)$ 's and $m_{0}$ for the number

of $\phi(0)$ 's. Here $\phi_{\delta_{j}}(m)$ is the spin-1 wave function introduced in Section 3. The second sum above stands for the sum of all distinct permutations of the $\phi_{\delta_{j}}$ 's.

The possible $m_{ \pm}$and $m_{0}$ are restricted by

$$
J=m_{+}+m_{0}+m_{-}, \quad m=m_{+}-m_{-}, \quad m_{+} \geq m_{-}
$$

such that

$$
2 m_{ \pm}=J \pm m-m_{0}
$$

$m_{0}$ ranges from $0(1), 2(3), \cdots$, to $J-m=$ even(odd). Since $\phi_{\delta_{1} \cdots \delta_{J}}$ does not depend on $\kappa$, one can choose $\kappa=0(1)$ for $J-m=$ even(odd), such that the coefficients under the sum on $m_{0}$ do not have square-root factors in them.

The wave functions for $J=2$ are

$$
\begin{aligned}
\phi_{\alpha \beta}(22) & =\phi_{\alpha}(+) \phi_{\beta}(+) \\
\sqrt{2} \phi_{\alpha \beta}(21) & =\phi_{\alpha}(+) \phi_{\beta}(0)+\phi_{\alpha}(0) \phi_{\beta}(+) \\
\sqrt{6} \phi_{\alpha \beta}(20) & =\phi_{\alpha}(+) \phi_{\beta}(-)+\phi_{\alpha}(-) \phi_{\beta}(+)+2 \phi_{\alpha}(0) \phi_{\beta}(0)
\end{aligned}
$$

and, for $J=3$,

$$
\begin{aligned}
& \phi_{\alpha \beta \gamma}(33)=\phi_{\alpha}(+) \phi_{\beta}(+) \phi_{\gamma}(+) \\
& \sqrt{3} \phi_{\alpha \beta \gamma}(32)=\phi_{\alpha}(+) \phi_{\beta}(+) \phi_{\gamma}(0)+\phi_{\alpha}(+) \phi_{\beta}(0) \phi_{\gamma}(+)+\phi_{\alpha}(0) \phi_{\beta}(+) \phi_{\gamma}(+) \\
& \sqrt{15} \phi_{\alpha \beta \gamma}(31)=\phi_{\alpha}(+) \phi_{\beta}(+) \phi_{\gamma}(-)+\phi_{\alpha}(+) \phi_{\beta}(-) \phi_{\gamma}(+)+\phi_{\alpha}(-) \phi_{\beta}(+) \phi_{\gamma}(+) \\
&+2\left[\phi_{\alpha}(+) \phi_{\beta}(0) \phi_{\gamma}(0)+\phi_{\alpha}(0) \phi_{\beta}(+) \phi_{\gamma}(0)+\phi_{\alpha}(0) \phi_{\beta}(0) \phi_{\gamma}(+)\right] \\
& \sqrt{10} \phi_{\alpha \beta \gamma}(30)=\phi_{\alpha}(0) \phi_{\beta}(+) \phi_{\gamma}(-)+\phi_{\alpha}(0) \phi_{\beta}(-) \phi_{\gamma}(+)+\phi_{\alpha}(-) \phi_{\beta}(+) \phi_{\gamma}(0) \\
&+ \phi_{\alpha}(+) \phi_{\beta}(-) \phi_{\gamma}(0)+\phi_{\alpha}(+) \phi_{\beta}(0) \phi_{\gamma}(-)+\phi_{\alpha}(-) \phi_{\beta}(0) \phi_{\gamma}(+) \\
&+2 \phi_{\alpha}(0) \phi_{\beta}(0) \phi_{\gamma}(0)
\end{aligned}
$$


For the case $m<0$, the wave functions can be obtained by using

$$
\phi(J-m)=(-)^{m} \phi^{*}(J m)
$$

In the $J \mathrm{RF}$, the wave functions $\phi(J m)$ have a simple form, i.e. the time components are zero as shown in the previous section for $J=1$. The wave functions for $s=1$ and $\sigma=1$ have been given in (3.2). So the wave functions for arbitrary spins $s$ and $\sigma$ are given again by (4.1), in which the spin-1 wave functions $\phi_{\alpha}(m)$ 's within the sum $\sum_{P}$ have been replaced by $\omega_{\alpha}(m)$ 's and $\varepsilon_{\alpha}(m)$ 's, respectively. Note that the general wave functions $\omega_{\alpha}(\mathrm{s} \mathrm{m})$ and $\varepsilon_{\alpha}(\sigma m)$, where $s$ and $\sigma$ are arbitrary integer, have nonzero components for all of their Lorentz indices $0,1,2$ and 3.

We now turn to the treatment of orbital-angular momenta in our formulation. We have shown in (2.12b) that the state $\left|\ell m_{\ell}\right\rangle$ which describes the orbital-angular momentum $\ell$ acts like a state at rest in the JRF. It is clear, therefore, that the correct wave function for $\ell$ must be described by a symmetric and traceless tensor of rank $\ell$ whose space components correspond to the relative momentum $\vec{r}$ but with zero time-component in the JRF. There are, in fact, two wave functions with zero time components in the JRF; a rank- $J$ tensor $\phi^{*}(J m)$ in the initial state and one $\chi(\ell 0) r^{\ell}$ representing the orbital angular momentum $\ell$ in the final state. They obey, be definition, $p^{\mu} \phi_{\mu}^{*}(J m)=0$ and $p^{\mu} \chi_{\mu}(\ell 0)=0$. Since we deal exclusively with collinear vectors aligned along the $z$-axis, the spin projection of the wave function comes with a zero $z$-component only, reflecting the fact that orbital angular momentum must be perpendicular to the $z$-axis [see $(2.5 b)]$. So we are left with the task of evaluating, in the $J \mathrm{RF}$, a symmetric and traceless tensor[1]

$$
\chi_{i j k \cdots}(\ell 0)=\frac{(\ell !)^{2}}{(2 \ell) !} \sum_{m_{0}} 2^{\left(\ell+m_{0}\right) / 2} \sum_{P}[\underbrace{\chi(+) \cdots}_{m_{+}} \underbrace{\chi(0) \cdots}_{m_{0}} \underbrace{\chi(-) \cdots}_{m_{-}}]_{i j k}
$$

and $m_{0}=0(1), 2(3), 4(5), \ldots$ for $\ell=$ even (odd). It is clear that $\chi(\ell 0)$ is devoid of the coefficients with square-root factors in the sum. We find

$$
\begin{aligned}
\chi(0) & =(0,0,1), \quad \chi( \pm)=\mp \frac{1}{\sqrt{2}}(1, \pm i, 0) \\
3 \chi_{i j}(20) & =\chi_{i}(+) \chi_{j}(-)+\chi_{i}(-) \chi_{j}(+)+2 \chi_{i}(0) \chi_{j}(0) \\
5 \chi_{i j k}(30) & =\chi_{i}(0) \chi_{j}(+) \chi_{k}(-)+\chi_{i}(0) \chi_{j}(-) \chi_{k}(+) \\
& +\chi_{i}(+) \chi_{j}(0) \chi_{j}(-)+\chi_{i}(-) \chi_{j}(0) \chi_{k}(+) \\
& +\chi_{i}(+) \chi_{j}(-) \chi_{j}(0)+\chi_{i}(-) \chi_{j}(+) \chi_{k}(0)+2 \chi_{i}(0) \chi_{j}(0) \chi_{k}(0)
\end{aligned}
$$


and

$$
\begin{aligned}
35 \chi_{i j k l}(40) & =2 \sum_{P}^{6}\left[\chi_{i}(+) \chi_{j}(-) \chi_{k}(+) \chi_{l}(-)\right] \\
+ & 4 \sum_{P}^{12}\left[\chi_{i}(0) \chi_{j}(0) \chi_{k}(+) \chi_{l}(-)\right]+8 \chi_{i}(0) \chi_{j}(0) \chi_{k}(0) \chi_{l}(0) \\
63 \chi_{i j k l m}(50) & =2 \sum_{P}^{30}\left[\chi_{i}(0) \chi_{j}(+) \chi_{k}(-) \chi_{l}(+) \chi_{m}(-)\right] \\
+ & 4 \sum_{P}^{20}\left[\chi_{i}(0) \chi_{j}(0) \chi_{k}(0) \chi_{l}(+) \chi_{m}(-)\right]+8 \chi_{i}(0) \chi_{j}(0) \chi_{k}(0) \chi_{l}(0) \chi_{m}(0)
\end{aligned}
$$

It should be noted that $\chi(\ell 0)$ is given only in space indices 1,2 and 3. The orbital angular momentum in the $J \mathrm{RF}$ does not have time components, and therefore it is in fact proportional[1] to $\phi(J m)$ in the $J$ RF with $J=\ell$ and $m=0$

$$
\chi_{i j k \ldots} \ldots(\ell 0)=c_{\ell} \phi_{i j k \ldots}(\ell 0), \quad c_{\ell}=\left[\frac{2^{\ell}(\ell !)^{2}}{(2 \ell) !}\right]^{1 / 2}
$$

The coefficients $c_{\ell}$ for a few values of $\ell$ are

$$
c_{1}=1, \quad c_{2}=\sqrt{\frac{2}{3}}, \quad c_{3}=\sqrt{\frac{2}{5}}, \quad c_{4}=\sqrt{\frac{8}{35}}
$$

\section{$5 \quad$ Wave Functions of Total Intrinsic Spin $S$}

The wave function $\psi(s, \sigma ; S \delta)$ corresponds to the state of total intrinsic spin $S$, built out of the wave functions for $s$ and $\sigma$ which are given by the tensors of rank- $s$ and $-\sigma$, respectively. Hence, it is a tensor of rank $s+\sigma$, given by

$$
\psi(s, \sigma ; S \delta)=\sum_{m_{a} m_{b}}\left(s m_{a} \sigma m_{b} \mid S \delta\right) \omega\left(s m_{a}\right) \varepsilon\left(\sigma m_{b}\right)
$$

where we have suppressed tensor indices in $\psi, \omega$ and $\varepsilon$ [see (2.9)]. In addition, we have suppressed the momentum labels in the wave functions.

Consider now the special case $s=\sigma=1$. Then, the $\psi(s, \sigma ; S \delta)$ is a rank-2 tensor. In the $J$ RF, a state in total intrinsic spin $S$ is

$$
\begin{aligned}
\psi^{\mu \nu}\left(s, \sigma ; S m_{S}\right) & =\sum_{m_{1} m_{2}}\left(s m_{1} \sigma m_{2} \mid S m_{S}\right) \omega^{\mu}\left(m_{1}\right) \varepsilon^{\nu}\left(m_{2}\right) \\
\psi^{\alpha \beta}\left(s, \sigma ; S-m_{S}\right) & =(-)^{S-m_{s}} \psi^{\alpha \beta *}\left(s, \sigma ; S m_{S}\right)
\end{aligned}
$$


Under an arbitrary rotation $R^{S}(\phi, \theta, 0)$, the daughter states $s$ and $\sigma$ transform

$$
\begin{aligned}
& R^{S}(\phi, \theta, 0) \omega^{\mu}\left(m_{1}\right) \equiv\left[R^{S}(\phi, \theta, 0)\right]_{\alpha}^{\mu} \omega^{\alpha}\left(m_{1}\right)=\sum_{m_{1}^{\prime}} \omega^{\mu}\left(m_{1}^{\prime}\right) D_{m_{1}^{\prime} m_{1}}^{s}(\phi, \theta, 0) \\
& R^{S}(\phi, \theta, 0) \varepsilon^{\nu}\left(m_{2}\right) \equiv\left[R^{S}(\phi, \theta, 0)\right]_{\beta}^{\nu} \varepsilon^{\beta}\left(m_{2}\right)=\sum_{m_{2}^{\prime}} \omega^{\nu}\left(m_{2}^{\prime}\right) D_{m_{2}^{\prime} m_{2}}^{\sigma}(\phi, \theta, 0)
\end{aligned}
$$

so that

$$
R^{S}(\phi, \theta, 0) \psi^{\mu \nu}\left(s, \sigma ; S m_{S}\right)=\sum_{m_{s}^{\prime}} \psi^{\mu \nu}\left(s, \sigma ; S m_{s}^{\prime}\right) D_{m_{s}^{\prime} m_{s}}^{S}(\phi, \theta, 0)
$$

This shows in fact that $\psi^{\mu \nu}\left(s, \sigma ; S m_{S}\right)$ is in a state of total intrinsic spin $S$. It is useful to write down the wave functions explicitly

$$
\left\{\begin{array}{l}
\psi^{\alpha \beta}(s, \sigma ; 22)=\omega^{\alpha}(+) \varepsilon^{\beta}(+), \quad \lambda=-\nu=+1 \\
\psi^{\alpha \beta}(s, \sigma ; 21)=\frac{1}{\sqrt{2}}\left[\omega^{\alpha}(+) \varepsilon^{\beta}(0)+\omega^{\alpha}(0) \varepsilon^{\beta}(+)\right] \\
\psi^{\alpha \beta}(s, \sigma ; 20)=\frac{1}{\sqrt{6}}\left[\omega^{\alpha}(+) \varepsilon^{\beta}(-)+\omega^{\alpha}(-) \varepsilon^{\beta}(+)+2 \omega^{\alpha}(0) \varepsilon^{\beta}(0)\right] \\
\psi^{\alpha \beta}(s, \sigma ; 11)=\frac{1}{\sqrt{2}}\left[\omega^{\alpha}(+) \varepsilon^{\beta}(0)-\omega^{\alpha}(0) \varepsilon^{\beta}(+)\right] \\
\psi^{\alpha \beta}(s, \sigma ; 10)=\frac{1}{\sqrt{2}}\left[\omega^{\alpha}(+) \varepsilon^{\beta}(-)-\omega^{\alpha}(-) \varepsilon^{\beta}(+)\right] \\
\psi^{\alpha \beta}(s, \sigma ; 00)=\frac{1}{\sqrt{3}}\left[\omega^{\alpha}(+) \varepsilon^{\beta}(-)+\omega^{\alpha}(-) \varepsilon^{\beta}(+)-\omega^{\alpha}(0) \varepsilon^{\beta}(0)\right]
\end{array}\right.
$$

In applications involving $\psi^{\mu \nu}\left(s, \sigma ; S m_{S}\right)$ listed above, we can safely ignore the non-zero time components in the wave functions $\omega^{\alpha}\left(m_{s}\right)$ and $\varepsilon^{\beta}\left(m_{\sigma}\right)$, because they are generally mated with $\chi(\ell 0)$ and $\phi^{*}(m)$ which have no time components in the $J$ RF. The sole exception occurs in the treatment of the decay $0^{+} \rightarrow 1^{-}+1^{-}$, where the non-zero time components of $\psi^{\mu \nu}\left(s, \sigma ; S m_{S}\right)$ could possibly enter; this case is considered in detail in the next section. See also Appendix $\mathrm{C}$ for additional decay amplitudes which involve the time components of $\psi^{\mu \nu}\left(s, \sigma ; S m_{S}\right)$ but not included in the main text.

\section{Covariant Helicity-Coupling Amplitudes}

The amplitude for $J \rightarrow s+\sigma$ in the $J$ RF is, in the helicity formalism [3],

$$
\mathcal{M}_{\lambda \nu}^{J}(M ; \theta, \phi)=\sqrt{\frac{2 J+1}{4 \pi}} F_{\lambda \nu}^{J} D_{M \delta}^{J *}(\phi, \theta, 0), \quad \delta=\lambda-\nu
$$


where $F_{\lambda \nu}^{J}$ is the helicity-coupling amplitude given by

$$
F_{\lambda \nu}^{J}=\sum_{\ell S}\left(\frac{2 \ell+1}{2 J+1}\right)^{1 / 2}(\ell 0 S \delta \mid J \delta)(s \lambda \sigma-\nu \mid S \delta) G_{\ell S}^{J} r^{\ell}
$$

Here $G_{\ell S}^{J}$ is the $\ell S$-coupling amplitude for which $\ell$ is the orbital angular momentum and $S$ is the total intrinsic spin, and $r^{\ell}$ is the barrier factor. The variable $r$ (see Table I) is measured in units of the scale factor $r_{0}$ (e.g. $1 \mathrm{fm}$ ), so should be understood as (unit-free) quantity $\left(r / r_{0}\right)^{\ell}$. For further comments on this point, see Conclusions and Discussions. Note that the rotationally invariant quantum numbers are given as super- and sub-scripts in the amplitude $\mathcal{M}_{\lambda \nu}^{J}, F_{\lambda \nu}^{J}$ and $G_{\ell S}^{J}$. Note that we must in principle include the spins $s$ and $\sigma$ as well in the amplitudes, i.e. the superscript $J$ should be replaced by $\{J s \sigma\}$. However, we shall continue to use simply the superscript $J$ for brevity of notation. The number of independent $F_{\lambda \nu}^{J}$ 's is the same as that of $G_{\ell S}^{J}$, as each of them describes the degrees of freedom for the decay process in the helicity and the canonical formalisms. For a general description of the number of independent $F_{\lambda \nu}^{J}$ 's, see ref. [1].

The coefficients of $G_{\ell S}^{J} r^{\ell}$ in (6.2) satisfy

$$
\begin{gathered}
\sum_{\ell S}\left(\frac{2 \ell+1}{2 J+1}\right)(\ell 0 S \delta \mid J \delta)^{2}(s \lambda \sigma-\nu \mid S \delta)^{2}=1 \quad \text { for a given }\{\lambda, \nu\} \\
\sum_{\lambda \nu}\left(\frac{2 \ell+1}{2 J+1}\right)^{1 / 2}(\ell 0 S \delta \mid J \delta)(s \lambda \sigma-\nu \mid S \delta)\left(\frac{2 \ell^{\prime}+1}{2 J+1}\right)^{1 / 2}\left(\ell^{\prime} 0 S^{\prime} \delta \mid J \delta\right)\left(s \lambda \sigma-\nu \mid S^{\prime} \delta\right) \\
=\delta_{\ell \ell^{\prime}} \delta_{S S^{\prime}} \quad \text { for a given }\left\{\ell, S, \ell^{\prime}, S^{\prime}\right\}
\end{gathered}
$$

with the proviso that $\ell$ and $\ell^{\prime}$ can be either always even or always odd, from parity conservation in the decay $J \rightarrow s+\sigma$. The relationship (6.3a) shows that the sum over a set $\{\lambda, \nu\}$ of the squares of all the coefficients of a given $G_{\ell S}^{J} r^{\ell}$ in (6.2) should be equal to one. The relationship (6.3b) for $\ell^{\prime}=\ell$ and $S^{\prime}=S$ can be used to show that the sum over the squares of the coefficients of $G_{\ell S}^{J} r^{\ell}$ in a given $F_{\lambda \nu}^{J}$ in (6.2) must be equal to one. We will show later with a number of simple examples how these constraints are borne out. From (6.3b) we obtain

$$
\sum_{\lambda \nu}\left|F_{\lambda \nu}^{J}\right|^{2}=\sum_{\ell S}\left|G_{\ell S}^{J} r^{\ell}\right|^{2}
$$

This simply shows that probability is conserved for both canonical and helicity description for the decay process $J \rightarrow s+\sigma$. 
Consider now $\theta=\phi=0$. The $D$-function in (6.1) is zero unless $M=\delta$. Hence we can write

$$
F_{\lambda \nu}^{J}=\sqrt{\frac{4 \pi}{2 J+1}} \mathcal{M}_{\lambda \nu}^{J}(\delta ; 0,0)
$$

So the helicity-coupling amplitude is proportional to the decay amplitude itself in which the the decay products $s$ and $\sigma$ are both aligned along the $z$-axis, in a coordinate system fixed in the $J$ RF. Hence, the momenta $\boldsymbol{q}$ and $\boldsymbol{k}$ have zero $x$ and $y$ components; see (3.1).

We now transform the helicity-coupling amplitude (6.2) by replacing, formally, the factor

$$
\left(\frac{2 \ell+1}{2 J+1}\right)^{1 / 2}(\ell 0 S \delta \mid J \delta) G_{\ell S}^{J} r^{\ell}
$$

with that proportional to a decay amplitude and write

$$
F_{\lambda \nu}^{J}=\sum_{\ell S}(s \lambda \sigma-\nu \mid S \delta) g_{\ell S}^{J} A_{\ell S}^{J}(s, \sigma ; \delta) r^{\ell}
$$

We assume that $g_{\ell S}^{J}$ is a constant to be measured experimentally, while $A_{\ell S}^{J}(s, \sigma ; \delta)$ is a covariant decay amplitude corresponding to the transition $|J \delta\rangle \rightarrow|S \delta\rangle+|\ell 0\rangle$ in which the decay products are aligned along the z-axis. The precise relationship between $g_{\ell S}^{J}$ and $G_{\ell S}^{J}$ will be given at the end of this section. It turns out that in general, there is more than one relativistic structure for a given $\{\ell S\}$. This is a consequence of treating $\gamma_{s}$ and $\gamma_{\sigma}$ as free parameters, which appear as factors in the relativistic terms related to a non-relativistic $G_{\ell S}^{J}$. This dependency on the particle masses can be disentangled if the widths of the daughter particles are sufficiently wide and the distribution function is examined over a sufficiently wide range of the parent mass.

The procedure for constructing covariant amplitudes goes as follows. To guarantee Lorentz invariance, one utilizes two invariant tensors of the Poincaré group[7], i.e.

$$
\begin{aligned}
g^{\mu \nu} & =g^{\alpha \beta} \Lambda_{\alpha}{ }^{\mu} \Lambda_{\beta}{ }^{\nu} \\
\varepsilon^{\nu \rho \sigma \tau} & =\varepsilon^{\mu \alpha \beta \gamma} \Lambda_{\mu}{ }^{\nu} \Lambda_{\alpha}{ }^{\rho} \Lambda_{\beta}{ }^{\sigma} \Lambda_{\gamma}{ }^{\tau}
\end{aligned}
$$

where $\Lambda_{\alpha}{ }^{\mu}$ is an element of the homogeneous Lorentz group (see also Appendix A). Now, we adopt a more restrictive procedure to ensure that the resulting amplitudes have a transparent transition to non-relativistic amplitudes. For the purpose, we write, in the $J$ RF, Lorentzinvariant decay amplitudes in the $\ell S$ coupling scheme via

$$
A_{\ell S}^{J}(s, \sigma ; \delta)=\left[p^{n_{0}}, \psi(s, \sigma ; S \delta), \chi(\ell 0), \phi^{*}(J \delta)\right]_{w}
$$


where $n_{0}=0$ or 1 and the notation $[\cdots]_{w}$ stands for Lorentz contraction of all the relevant Lorentz indices in the $J$ RF. We adopt the following two rules:

(a) with the modified metric $\tilde{g}(w)[$ see (3.4) $]$ and only with this metric, if $s+\sigma+$ $\ell-J=$ even. Here $p^{\mu}$ never appears in the amplitudes, i.e. $n_{0}=0$.

(b) with the totally antisymmetric rank-4 tensor $\left(\epsilon^{\alpha \beta \mu \nu}\right)$ together with the 4momentum $p^{\mu}$, and with $\tilde{g}(w)$ whenever necessary, if $s+\sigma+\ell-J=$ odd. We restrict the use of $\epsilon^{\alpha \beta \mu \nu}$ and $p^{\mu}$ once and only once, so that $n_{0}=1$.

(c) For angular momentum $\ell$, only amplitudes proportional to $r^{\ell}$ are considered $\left(r^{\ell}\right.$-rule).

It is seen that $p^{\mu}=(w ; 0,0,0)$ is used in two different ways, once inside the definition of $\tilde{g}(w)$, and again with the rank-4 tensor if necessary. Since collinear vectors are used in $A_{\ell S}^{J}(s, \sigma ; \delta)$, it cannot give angular dependence; but instead it gives dependence on the Lorentz factors $\gamma_{s}$ and $\gamma_{\sigma}$-which is the reason for calculating the invariant amplitudes in this manner. The decay amplitudes which lie beyond the scope of the rules above, are treated in Appendix C, and the reasons explained for not including in the examples given in this paper.

It will be shown later that the presence of the rank-4 tensor along with $p$ in the amplitude (6.9) leads to a factor $(i w)$ when evaluated in the $J$ RF. As the wave functions which appear in (6.9) are all unitless, we can impose the unitless condition in (6.9) by substiuting $p^{n_{0}}$ by $\left[p /\left(i w_{\text {th }}\right)\right]^{n_{0}}$ where $w_{\text {th }}$ is the $w$ at its threshold. So the factor $(i w)$ which appears the amplitudes is then replaced by $\left(w / w_{\text {th }}\right)$. However, we will continue to use $p^{n_{0}}$ and the factor $(i w)$ in this paper for simplicity of notation. The reader will note that the situation here is similar to that for $r^{\ell}$ against $\left(r / r_{0}\right)^{\ell}$ commented on previously in this section. It is perhaps more appropriate to write the covariant amplitudes as

$$
A_{\ell S}^{J}(s, \sigma ; \delta)=\left[\left(\frac{p}{i w_{\mathrm{th}}}\right)^{n_{0}}, \psi(s, \sigma ; S \delta), \chi(\ell 0), \phi^{*}(J \delta)\right]_{w}
$$

Note that the right-hand side is now explicitly unitless. We will come back to this point again in Conclusions and Discussions.

The wave function $\phi^{*}(J \delta)$ refers to the initial spin $J$ and $\chi(\ell 0)$ to the orbital angular momentum $\ell$ in the final state. They are $\operatorname{rank}-J$ and rank- $\ell$ tensors, respectively. The wave functions of total intrinsic spin $S$, denoted by $\psi(s, \sigma ; S \delta)$ in (6.9), has been given in (5.1). 
It is a tensor of rank $s+\sigma$ built out of $\omega$ 's and $\varepsilon$ 's. For the different possible Lorentz contractions, it is relevant how many indices of the $\omega$ 's and $\varepsilon$ 's are contracted with $\phi$ and $\chi$. For the purpose, it is necessary to introduce a new notation, in order to uniquely specify the process of Lorentz contraction. We transform $\psi$ to $\Psi$ as follows:

$$
\psi\left(s, \sigma ; S m_{S}\right) \quad \longrightarrow \quad \Psi\left(n_{1}, n_{2} ; S m_{S}\right)
$$

where

$$
n_{1}=n_{\omega \phi}, \quad n_{1}^{\prime}=n_{\omega \phi}+1, \quad n_{2}=n_{\varepsilon \phi}, \quad n_{2}^{\prime}=n_{\varepsilon \phi}+1
$$

Here $n_{\omega \phi}\left(n_{\varepsilon \phi}\right)$ is the number of contractions of $\phi$ with $\omega(\varepsilon)$. The reason for a change of notation from $\psi$ to $\Psi$ is to merely indicate that the variables $\{s, \sigma\}$ of $\psi$ have been changed to $\left\{n_{1}, n_{2}\right\}$. The meaning of apostrophes is explained shortly. The remaining indices of $\omega$ and $\varepsilon$ are contracted with $\chi$ or among themselves, except that, if necessary, three indices from $\omega$, $\varepsilon, \chi$ and $\phi$ (one and only one index from each wave function for a total of three), along with $p^{\mu}$, are contracted with the totally antisymmetric rank-4 tensor $\epsilon^{\alpha \beta \mu \nu}$. This procedure is illustrated in Tables II through IV; The heading for the column representing $\Psi\left(n_{1}, n_{2} ; S m_{S}\right)$ is simply written as $R\left(n_{1}\right)$ if $s \geq 1$ and $\sigma=0$ or $R\left(n_{1}, n_{2}\right)$ if $s \geq 1$ and $\sigma \geq 1$, where $R=s+\sigma$ is the rank of the tensor $\psi$. One or two dots above $R$, i.e. $\dot{R}$ or $\ddot{R}$, indicate one or two internal contractions between $\omega$ and $\varepsilon$. The number of dots between the the $\psi, \chi$ and $\phi$ indicate the number of indices to be contracted between neighbors. If the contraction involving $\epsilon^{\alpha \beta \mu \nu}$ appears, the appropriate indices are indicated by apostrophes (there are always three and only three apostrophes for each amplitude).

The helicity-coupling amplitudes satisfy, from parity conservation in the strong decay,

$$
F_{\lambda \nu}^{J}=\eta_{J} \eta_{s} \eta_{\sigma}(-)^{J-s-\sigma} F_{-\lambda-\nu}^{J}=(-)^{s+\sigma+\ell-J} F_{-\lambda-\nu}^{J}
$$

where the product of intrinsic parities is linked to the allowed orbital angular momenta via $\eta_{J} \eta_{s} \eta_{\sigma}=(-)^{\ell}$, so for a given decay channel either $F_{\lambda \nu}^{J}=F_{-\lambda-\nu}^{J}$ or $F_{\lambda \nu}^{J}=-F_{-\lambda-\nu}^{J}$ holds for all amplitudes. From (6.7), we see that

$$
A_{\ell S}^{J}(s, \sigma ; \delta)=(-)^{\ell+S-J} A_{\ell S}^{J}(s, \sigma ;-\delta)
$$

If particles $s$ and $\sigma$ are identical, we have[8, 3]

$$
F_{\lambda \nu}^{J}(s, \sigma)=(-)^{J} F_{\nu \lambda}^{J}(\sigma, s)
$$


and this leads to, from (6.7),

$$
A_{\ell S}^{J}(s, \sigma ; \delta)=(-)^{J} A_{\ell S}^{J}(\sigma, s ;-\delta)
$$

We impose the identical-particle condition by substituting

$$
\begin{aligned}
F_{\lambda \nu}^{J}(s, \sigma) & \rightarrow \frac{1}{2}\left[F_{\lambda \nu}^{J}(s, \sigma)+(-)^{J} F_{\nu \lambda}^{J}(\sigma, s)\right] \\
A_{\ell S}^{J}(s, \sigma ; \delta) & \rightarrow \frac{1}{2}\left[A_{\ell S}^{J}(s, \sigma ; \delta)+(-)^{J} A_{\ell S}^{J}(\sigma, s ;-\delta)\right]
\end{aligned}
$$

In this operation, we need to point out that the factor $r^{\ell}$ in (6.7) must remain invariant; in another words, ' $r$ ' stands for the magnitude of ' $r$ ' and hence it does not change sign.

The formulas (6.7) and (6.9) constitute the main result of this paper. We give a few examples of practical importance to illustrate the use of these formulas. For the purpose, the following general formulas will prove to be useful. Observe

$$
\begin{aligned}
\phi^{*}(J m) & =(-)^{m} \phi(J-m) \\
\omega^{*}\left(s m_{s}\right) & =(-)^{m_{s}} \omega\left(s-m_{s}\right), \quad \varepsilon^{*}\left(\sigma m_{\sigma}\right)=(-)^{m_{\sigma}} \varepsilon\left(\sigma-m_{\sigma}\right) \\
\chi^{*}(\ell 0) & =\chi(\ell 0)
\end{aligned}
$$

so we find

$$
\psi(s, \sigma ; S-\delta)=(-)^{s+\sigma-S}(-)^{\delta} \psi^{*}(s, \sigma ; S \delta)
$$

and

$$
\begin{aligned}
A_{\ell S}^{J}(s, \sigma ;-\delta) & =\left[p, \psi(s, \sigma ; S-\delta), \chi(\ell 0), \phi^{*}(J-\delta)\right]_{w} \\
& =(-)^{s+\sigma-S} A_{\ell S}^{J *}(s, \sigma ; \delta)
\end{aligned}
$$

and so, combining with (6.14),

$$
A_{\ell S}^{J *}(s, \sigma ; \delta)=(-)^{s+\sigma+\ell-J} A_{\ell S}^{J}(s, \sigma ; \delta)
$$

So we see that $A_{\ell S}^{J}(s, \sigma ; \delta)$ is purely real (imaginary) if $s+\sigma+\ell-J=$ even (odd). We see that this is consistent with (6.9): the amplitudes $A_{\ell S}^{J}(s, \sigma ; \delta)$ are real in general, except when the $\epsilon^{\alpha \beta \gamma \delta}$ and $p$ are used to form a Lorentz scalar, in which case the amplitudes are proportional to $(i w)$ times a real factor, so that they become purely imaginary. Since $(\ell 0 S \delta \mid J \delta)$ is contained in $A_{\ell S}^{J}(s, \sigma ; \delta)$, we must have

$$
A_{\ell S}^{J}(s, \sigma ; 0)=0, \quad \text { if } \quad \ell+S-J=\text { odd }
$$


If there are more than one amplitude for a given $\{\ell S\}$, then we need to include them all by modifying the appropriate factor in (6.7)

$$
g_{\ell S}^{J} A_{\ell S}^{J}(s, \sigma ; \delta) \Rightarrow \sum_{i}{ }^{(i)} g_{\ell S}^{J}{ }^{(i)} A_{\ell S}^{J}(s, \sigma ; \delta)
$$

where ${ }^{(i)} g_{\ell S}^{J}$ 's are now the phenomenological constants to be determined experimentally. In the examples given in this paper, there are no more than three different amplitudes for a given $\{\ell S\}$; so for a more transparent notation we denote the amplitudes $A, B$ and $C$, with the constants $g, f$ and $h$, respectively.

Let $\xi(m), \xi^{\prime}(m)$ and $\xi^{\prime \prime}(m)$ stand for any of the wave functions $\omega, \varepsilon, \chi$ or $\phi$. For the calculation of the amplitudes it proves useful to note that

$$
\left\{\begin{aligned}
\boldsymbol{\xi}^{*}( \pm) & =-\boldsymbol{\xi}(\mp), \quad \boldsymbol{\xi}^{*}(0)=\boldsymbol{\xi}(0) \\
\boldsymbol{\xi}^{*}(m) \cdot \boldsymbol{\xi}^{\prime}\left(m^{\prime}\right) & =\delta_{m m^{\prime}}, \quad \text { if } m \text { or } m^{\prime}= \pm 1 \\
\boldsymbol{\xi}^{*}(0) \cdot \boldsymbol{\xi}^{\prime}(0) & =1, \quad \text { if } \xi \text { and } \xi^{\prime}=\chi \text { or } \phi \\
\boldsymbol{\xi}^{*}(0) \cdot \boldsymbol{\xi}^{\prime}(0) & =\gamma_{s}, \quad \text { if } \xi \text { or } \xi^{\prime}=\omega \text { and the other }=\chi \text { or } \phi \\
\boldsymbol{\xi}^{*}(0) \cdot \boldsymbol{\xi}^{\prime}(0) & =\gamma_{\sigma}, \quad \text { if } \xi \text { or } \xi^{\prime}=\varepsilon \text { and the other }=\chi \text { or } \phi \\
\omega^{*}(0) \cdot \varepsilon(0) & =\omega(0) \cdot \varepsilon^{*}(0)=\omega(0) \cdot \varepsilon(0)=\gamma_{s} \gamma_{\sigma}
\end{aligned}\right.
$$

and also, using a short-hand notation where $\xi, \xi^{\prime}, \xi^{\prime \prime}$ follow the sequence $\omega, \varepsilon, \chi, \phi$,

$$
\left[(m)\left(m^{\prime}\right)\left(m^{\prime \prime}\right)\right]=\left[p, \xi(m), \xi^{\prime}\left(m^{\prime}\right), \xi^{\prime \prime} *\left(m^{\prime \prime}\right)\right]=0
$$

except the following:

$$
\left\{\begin{array}{l}
{[( \pm)(\mp)(0)]= \pm(i w) a} \\
{[( \pm)(0)( \pm)]= \pm(i w) a} \\
{[(0)( \pm)( \pm)]=\mp(i w) a}
\end{array}\right.
$$

where

$$
\begin{cases}a=1, & \text { if }(0)=\chi(0) \text { or } \phi(0) \\ a=\gamma_{s}, & \text { if }(0)=\omega(0) \\ a=\gamma_{\sigma}, & \text { if }(0)=\varepsilon(0)\end{cases}
$$

We are now ready to give the non-relativistic $G_{\ell s}^{J}$ 's in terms of the relativistic $g_{\ell s}^{J}$ 's. From 
(6.6), (6.7) and (6.23), we find

$$
\begin{aligned}
G_{\ell S}^{J}=\left(\frac{2 \ell+1}{2 J+1}\right)^{-1 / 2}(\ell 0 S \delta \mid J \delta)^{-1} & \\
& \times\left.\sum_{i}{ }^{(i)} g_{\ell S}^{J}{ }^{(i)} A_{\ell S}^{J}(s, \sigma ; \delta)\right|_{\left(w=w_{\text {th }} \text { and } \gamma_{s}=\gamma_{\sigma}=1\right)}
\end{aligned}
$$

where $w_{\text {th }}$ is the $w$ at its threshold. We emphasize here that our decay amplitudes, constructed with the prescription following ([6.9), correspond to the process $|J m\rangle \rightarrow|S \delta\rangle+|\ell 0\rangle$ and hence it must be proportional to the Clebsch-Gordan coefficient $(\ell 0 S \delta \mid J \delta)$. What is remarkable is that the proportionality constant is independent of $\delta$ in the nonrelativistic limit, i.e. $\gamma_{s}=\gamma_{\sigma}=1$ and $w=w_{\mathrm{th}}$. This result can be traced to the fact that the wave functions $\phi^{*}(J \delta)$ and $\chi(\ell 0) r^{\ell}$ are at rest in the $J \mathrm{RF}$, so that they do not have time components. So, even though the wave functions $\phi(s, \sigma ; S \delta)$ with a total intrinsic spin $S$ have nonzero time components, they do not contribute to the covariant decay amplitudes (6.9) and (6.10). The resulting amplitudes are proportional to $\left(w / w_{\text {th }}\right)^{n_{0}}$ with $n_{0}(=0,1)$ defined according to the prescription following (6.9) and are functions of $\gamma_{s}$ and $\gamma_{\sigma}$ only. As a consequence, our invariant amplitudes lead to the nonrelativistic helicity-coupling amplitude $F_{\lambda \nu}^{J}$ of (6.2) through the formula (6.28).

We note that, if the Clebsch-Gordan coefficient in the denominator in (6.28) is zero, the amplitude $A_{\ell S}^{J}(s, \sigma ; \delta)$ is zero, and hence $g_{\ell s}^{J}$ and $G_{\ell s}^{J}$ can be set to zero as well. From (6.9), we see that $G_{\ell s}^{J}$ 's are proportional to a factor $\left(i w_{\text {th }}\right)$ if $s+\sigma+\ell-J=$ odd, but it is absent if $s+\sigma+\ell-J=$ even. If we had used the unitless prescription as given (6.10), then the factor $\left(i w_{\mathrm{th}}\right)$ would have been replaced by $\left(w / w_{\mathrm{th}}\right)$. See the section on Conclusions and Discussions for further comments on (6.28).

Finally, we wish to point out that there exists one important class of decay modes for which there is just one intermediate state with nonzero spin. Specifically, let $\sigma=\nu=0$. Then we have $S=s, \delta=\lambda$ and $\psi(s, \sigma ; S \delta)=\omega(s \delta)$, so that

$$
\begin{aligned}
F_{\delta}^{J} & =\sum_{\ell} g_{\ell s}^{J} A_{\ell s}^{J}(s, \sigma ; \delta) r^{\ell} \\
A_{\ell s}^{J}(s, \sigma ; \delta) & =\left[p^{n_{0}}, \omega(s \delta), \chi(\ell 0), \phi^{*}(J \delta)\right]_{w}, \quad n_{0}=0,1
\end{aligned}
$$

Here the invariant amplitude refers to the transition $|J \delta\rangle \rightarrow|s \delta\rangle+|\ell 0\rangle$. The helicity-coupling amplitudes (6.2) reduce to

$$
F_{\delta}^{J}=\sum_{\ell}\left(\frac{2 \ell+1}{2 J+1}\right)^{1 / 2}(\ell 0 s \delta \mid J \delta) G_{\ell s}^{J} r^{\ell}
$$


The formula (6.28) for this case reduces to

$$
G_{\ell s}^{J}=\left.\left(\frac{2 \ell+1}{2 J+1}\right)^{-1 / 2}(\ell 0 s \delta \mid J \delta)^{-1} g_{\ell s}^{J} A_{\ell s}^{J}(s, \sigma ; \delta)\right|_{\left(w=w_{\text {th }} \text { and } \gamma_{s}=1\right)}
$$

It can be shown that there exists a single $A_{\ell s}^{J}(s, \sigma ; \delta)$ for any given set $\{J, \ell, s\}$, i.e. the summation on $i$ in (6.28) does not apply here. Once again, the right-hand side of the formula above does not depend on $\delta$, because its dependence on the amplitude $A_{\ell s}^{J}(s, \sigma ; \delta)$ is cancelled by the Clebsch-Gordan coefficient in the denominator.

\section{Illustrative Examples I}

Here we give a set of selected examples to illustrate the principles involved and to demonstrate the need to carefully examine each, so as to cast the invariant amplitudes as general as feasible. We confine ourselves to the cases in which both of the decay products have spins given by $s=1$ or 2 and $\sigma=0$. Although the decay modes in which one of the two decay products is spinless have already been treated adequately in a previous paper[1], we start with three such examples in order to contrast with those treated in Appendix C. A nontrivial case with $J=3$ is treated as a fourth example.

We first give in Tables IIa and IIb the decay amplitudes with $s=1$ or 2 and $\sigma=0$ and then give $F^{J}$ 's explicitly with four examples. 
Table IIa: Decay Amplitudes

for $s+\ell-J=$ even

$(s=1$ or 2 and $\sigma=0)$

$R$ stands for the rank.

\begin{tabular}{|c|c|c|c|c|c|}
\hline & $R_{s}\left(n_{1}\right)$ & & $R_{\ell}$ & & $R_{J}$ \\
\hline 1 & $1(0)$ & . & 1 & & 0 \\
\hline 2 & $1(1)$ & & 0 & & 1 \\
\hline 3 & $1(0)$ & . & 2 & . & 1 \\
\hline 4 & $1(1)$ & & 1 & . & 2 \\
\hline 5 & $1(0)$ & . & 3 & $:$ & 2 \\
\hline 6 & $1(1)$ & & 2 & $:$ & 3 \\
\hline 7 & $1(0)$ & . & 4 & $: \cdot$ & 3 \\
\hline 8 & $1(1)$ & & 3 & $\cdot:$ & 4 \\
\hline 9 & $1(1)$ & $\cdot$ & 5 & $::$ & 4 \\
\hline 10 & $1(1)$ & & 4 & $::$ & 5 \\
\hline 11 & $1(0)$ & . & 6 & $::$ & 5 \\
\hline 1 & $2(0)$ & : & 2 & & 0 \\
\hline 2 & $2(1)$ & . & 1 & & 1 \\
\hline
\end{tabular}

Table IIb: Decay Amplitudes

for $s+\ell-J=$ even

$(s=1$ or 2 and $\sigma=0)$

$R$ stands for the rank.

$R_{s}\left(n_{1}\right) \quad R_{\ell} \quad R_{J}$

\begin{tabular}{|c|c|c|c|c|c|}
\hline 3 & $2(0)$ & $:$ & 3 & . & 1 \\
\hline 4 & $2(2)$ & & 0 & & 2 \\
\hline 5 & $2(1)$ & . & 2 & . & 2 \\
\hline 6 & $2(0)$ & : & 4 & : & 2 \\
\hline 7 & $2(2)$ & & 1 & . & 3 \\
\hline 8 & $2(1)$ & . & 3 & : & 3 \\
\hline 9 & $2(0)$ & : & 5 & $:$ & 3 \\
\hline 10 & $2(2)$ & & 2 & : & 4 \\
\hline 11 & $2(1)$ & . & 4 & $\cdot:$ & 4 \\
\hline 12 & $2(0)$ & : & 6 & $::$ & 4 \\
\hline 13 & $2(2)$ & & 3 & $\cdot:$ & 5 \\
\hline 14 & $2(1)$ & . & 5 & $::$ & 5 \\
\hline 15 & $2(0)$ & : & 7 & $::$ & 5 \\
\hline
\end{tabular}


Table IIc: Decay Amplitudes

for $s+\ell-J=$ odd

$(s=1$ or 2 and $\sigma=0)$

$R$ stands for the rank.

\begin{tabular}{|l|llll|}
\multicolumn{2}{c}{$R_{s}\left(n_{1}\right)$} & $R_{\ell}$ & $R_{J}$ \\
\hline 1 & $1\left(1^{\prime}\right)$ & $1^{\prime}$ & & $1^{\prime}$ \\
2 & $1\left(1^{\prime}\right)$ & $2^{\prime}$ & $\cdot$ & $2^{\prime}$ \\
3 & $1\left(1^{\prime}\right)$ & $3^{\prime}$ & $:$ & $3^{\prime}$ \\
4 & $1\left(1^{\prime}\right)$ & $4^{\prime}$ & $: \cdot$ & $4^{\prime}$ \\
5 & $1\left(1^{\prime}\right)$ & $5^{\prime}$ & $::$ & $5^{\prime}$ \\
\hline 1 & $2\left(1^{\prime}\right)$ & $2^{\prime}$ & & $1^{\prime}$ \\
2 & $2\left(2^{\prime}\right)$ & $1^{\prime}$ & $2^{\prime}$ \\
\hline
\end{tabular}

Table IId: Decay Amplitudes

for $s+\ell-J=$ odd

$(s=1$ or 2 and $\sigma=0)$

$R$ stands for the rank.

$R_{s}\left(n_{1}\right) \quad R_{\ell} \quad R_{J}$

\begin{tabular}{|l|lllll|}
\hline 3 & $2\left(1^{\prime}\right)$ & $\cdot$ & $3^{\prime}$ & $\cdot$ & $2^{\prime}$ \\
4 & $2\left(2^{\prime}\right)$ & $2^{\prime}$ & $\cdot$ & $3^{\prime}$ \\
5 & $2\left(1^{\prime}\right)$ & $\cdot$ & $4^{\prime}$ & $:$ & $3^{\prime}$ \\
6 & $2\left(2^{\prime}\right)$ & & $3^{\prime}$ & $:$ & $4^{\prime}$ \\
7 & $2\left(1^{\prime}\right)$ & $\cdot$ & $5^{\prime}$ & $: \cdot$ & $4^{\prime}$ \\
8 & $2\left(2^{\prime}\right)$ & & $4^{\prime}$ & $:$ & $5^{\prime}$ \\
9 & $2\left(1^{\prime}\right)$ & $\cdot$ & $6^{\prime}$ & $::$ & $5^{\prime}$ \\
\hline
\end{tabular}

$7.1 \quad \underline{1^{-} \rightarrow 1^{-}+0^{-}}$

Here we must have $\ell=1$ from parity conservation, and the decay amplitude (6.9) in the $J \mathrm{RF}$ is

$$
\begin{aligned}
A_{11}^{(1)}(s, \sigma ; \delta)=\left[p, \omega(1 \delta), \chi(10), \phi^{*}(1 \delta)\right]_{w} & =\epsilon^{\alpha \beta \gamma \delta} p_{\alpha} \omega_{\beta}(1 \delta) \chi_{\gamma}(10) \phi_{\delta}^{*}(1 \delta) \\
& =w \epsilon_{i j k} \omega_{i}(1 \delta) \chi_{j}(10) \phi_{k}^{*}(1 \delta) \\
& = \pm(i w), \quad \delta= \pm 1 \\
& =0, \quad \delta=0
\end{aligned}
$$

The amplitude given here corresponds to row 1, Table IIc. Note that (7.1) is in fact the only acceptable decay amplitude which satisfies (6.13). The helicity-coupling amplitudes are, from (6.7),

$$
F_{ \pm}^{(1)}= \pm(i w) g_{11}^{(1)} r, \quad F_{0}^{(1)}=0
$$

where $g_{11}^{(1)}$, with $\{\ell S\}=\{11\}$, is an arbitrary phenomenological constant. In the nonrelativistic limit, we have $\sqrt{2} F_{ \pm}^{(1)}=\mp G_{11}^{(1)} r$ and $F_{0}^{(1)}=0$. So we conclude $G_{11}^{(1)}=$ $-\sqrt{2}(i w) g_{11}^{(1)}$ 
$7.2 \stackrel{2^{-} \rightarrow 1^{-}+0^{-}}{ }$

There are two orbital angular momenta $\ell=1$ and $\ell=3$. The covariant amplitudes are given in rows 4 and 5 , Table IIa, for pure $P$ - and $F$-waves, respectively. They are

$$
\begin{aligned}
A_{11}^{(2)}(s, \sigma ; \delta) & =\left[\omega(1 \delta), \chi(10), \phi^{*}(2 \delta)\right]_{w} \\
& =\omega^{\alpha}(1 \delta) \chi^{\beta}(10) \phi_{\alpha \beta}^{*}(2 \delta) \\
A_{31}^{(2)}(s, \sigma ; \delta) & =\left[\omega(1 \delta), \chi(30), \phi^{*}(2 \delta)\right]_{w} \\
& =\omega_{\alpha}(1 \delta) \chi^{\alpha \beta \gamma}(30) \phi_{\beta \gamma}^{*}(2 \delta)
\end{aligned}
$$

so that, using the Lorentz metric $\tilde{g}(w)$ in the $J$ RF,

$$
\begin{aligned}
& A_{11}^{(2)}(s, \sigma ; 1)=\frac{1}{\sqrt{2}}, \quad A_{11}^{(2)}(s, \sigma ; 0)=\sqrt{\frac{2}{3}} \gamma_{s} \\
& A_{31}^{(2)}(s, \sigma ; 1)=-\frac{\sqrt{2}}{5}, \quad A_{31}^{(2)}(s, \sigma ; 0)=\frac{\sqrt{6}}{5} \gamma_{s}
\end{aligned}
$$

From this one obtains

$$
\left\{\begin{array}{l}
F_{+}^{(2)}=\frac{1}{\sqrt{2}}\left(g_{11}^{(2)}-\frac{2}{5} g_{31}^{(2)} r^{2}\right) r \\
F_{0}^{(2)}=\sqrt{\frac{2}{3}} \gamma_{s}\left(g_{11}^{(2)}+\frac{3}{5} g_{31}^{(2)} r^{2}\right) r
\end{array}\right.
$$

The helicity-coupling amplitudes in the non-relativistic limit are, from (6.30),

$$
\left\{\begin{aligned}
\sqrt{2} F_{+}^{(2)} & =\sqrt{\frac{3}{5}} G_{11}^{(2)} r+\sqrt{\frac{2}{5}} G_{31}^{(2)} r^{3} \\
F_{0}^{(2)} & =\sqrt{\frac{2}{5}} G_{11}^{(2)} r-\sqrt{\frac{3}{5}} G_{31}^{(2)} r^{3}
\end{aligned}\right.
$$

We obtain, from (6.31),

$$
G_{11}^{(2)}=\sqrt{\frac{5}{3}} g_{11}^{(2)}, \quad G_{31}^{(2)}=-\sqrt{\frac{2}{5}} g_{31}^{(2)}
$$


$7.3 \quad \underline{1^{+} \rightarrow 2^{+}+0^{-}}$

This decay can proceed via two allowed values of $\ell$, i.e. $\ell=1$ or 3 (see rows 2 and 3 , table IIb).

$$
\begin{aligned}
A_{12}^{(1)}(s, \sigma ; \delta) & =\left[\omega(2 \delta), \chi(10), \phi^{*}(1 \delta)\right]_{w} \\
& =\omega^{\alpha \beta}(2 \delta) \chi_{\beta}(10) \phi_{\alpha}^{*}(1 \delta) \\
& =\frac{1}{\sqrt{2}} \gamma_{s}, \quad \delta=+1 \\
& =\sqrt{\frac{2}{3}} \gamma_{s}^{2}, \quad \delta=0
\end{aligned}
$$

and

$$
\begin{aligned}
A_{32}^{(1)}(s, \sigma ; \delta) & =\left[\omega(2 \delta), \chi(30), \phi^{*}(1 \delta)\right]_{w} \\
& =\omega_{\alpha \beta}(2 \delta) \chi^{\alpha \beta \gamma}(30) \phi_{\gamma}^{*}(1 \delta) \\
& =-\frac{\sqrt{2}}{5} \gamma_{s}, \quad \delta=+1 \\
& =\frac{1}{5} \sqrt{\frac{2}{3}}\left(1+2 \gamma_{s}^{2}\right), \quad \delta=0
\end{aligned}
$$

The helicity-coupling amplitudes are, from (6.7) with $J=1$,

$$
\left\{\begin{array}{l}
F_{+}^{(1)}=\frac{1}{\sqrt{2}}\left(g_{12}^{(1)} r-\frac{2}{5} g_{32}^{(1)} r^{3}\right) \gamma_{s} \\
F_{0}^{(1)}=\sqrt{\frac{2}{3}}\left[g_{12}^{(1)} \gamma_{s}^{2} r+\frac{3}{5} g_{32}^{(1)}\left(\frac{1}{3}+\frac{2}{3} \gamma_{s}^{2}\right) r^{3}\right]
\end{array}\right.
$$

where $g_{12}^{(1)}$ and $g_{32}^{(1)}$ are arbitrary phenomenological constants.

In the non-relativistic limit, we obtain

$$
\left\{\begin{aligned}
\sqrt{2} F_{+}^{(1)} & =-\sqrt{\frac{3}{5}} G_{12}^{(1)} r-\sqrt{\frac{2}{5}} G_{32}^{(1)} r^{3} \\
F_{0}^{(1)} & =-\sqrt{\frac{2}{5}} G_{12}^{(1)} r+\sqrt{\frac{3}{5}} G_{32}^{(1)} r^{3}
\end{aligned}\right.
$$

Here we see for the first time the rules $(\underline{6.3 a})$ and $(\underline{6.3 b})$ are obeyed by the formula above. Note also that (6.4) is satisfied as well. If we set $\gamma_{s}=1$ in (7.10), we find that

$$
G_{12}^{(1)}=-\sqrt{\frac{5}{3}} g_{12}^{(1)} \text { and } \quad G_{32}^{(1)}=\sqrt{\frac{2}{5}} g_{32}^{(1)}
$$

which can be obtained directly by applying (6.31). 


\section{$7.4 \quad \underline{3^{+} \rightarrow 2^{+}+0^{-}}$}

We give, as a final example of this section, the helicity-coupling amplitudes for $J=3$, $s=2$ and $\sigma=0$. The requisite amplitudes, $A_{\ell S}^{(3)}(s, \sigma ; \delta)$, require three orbital angular momenta $\ell=1,3$ and 5 , as given by rows 7, 8, and 9 in Table IIb. The results are

$$
\begin{aligned}
& A_{12}^{(3)}(s, \sigma ; 2)=\frac{1}{\sqrt{3}}, A_{12}^{(3)}(s, \sigma ; 1)=2 \sqrt{\frac{2}{15}} \gamma_{s}, A_{12}^{(3)}(s, \sigma ; 0)=\frac{1}{\sqrt{15}}\left(2 \gamma_{s}^{2}+1\right) \\
& A_{32}^{(3)}(s, \sigma ; 2)=-\frac{2}{5 \sqrt{3}}, A_{32}^{(3)}(s, \sigma ; 1)=\frac{1}{5} \sqrt{\frac{2}{15}} \gamma_{s}, A_{32}^{(3)}(s, \sigma ; 0)=\frac{2}{15} \sqrt{\frac{3}{5}}\left(3 \gamma_{s}^{2}-1\right) \\
& A_{52}^{(3)}(s, \sigma ; 2)=\frac{2}{21 \sqrt{3}}, A_{52}^{(3)}(s, \sigma ; 1)=-\frac{10}{21} \sqrt{\frac{2}{15}} \gamma_{s}, A_{52}^{(3)}(s, \sigma ; 0)=\frac{20}{63 \sqrt{15}}\left(2 \gamma_{s}^{2}+1\right)
\end{aligned}
$$

which lead to

$$
\left\{\begin{array}{l}
F_{2}^{(3)}=\frac{1}{\sqrt{3}}\left(g_{12}^{(3)}-\frac{2}{5} g_{32}^{(3)} r^{2}+\frac{2}{21} g_{52}^{(3)} r^{4}\right) r \\
F_{1}^{(3)}=\sqrt{\frac{2}{15}}\left(2 g_{12}^{(3)}+\frac{1}{5} g_{32}^{(3)} r^{2}-\frac{10}{21} g_{52}^{(3)} r^{4}\right) \gamma_{s} r \\
F_{0}^{(3)}=\sqrt{\frac{3}{5}}\left\{g_{12}^{(3)}\left(\frac{2}{3} \gamma_{s}^{2}+\frac{1}{3}\right)+\frac{4}{15} g_{32}^{(3)}\left(\frac{3}{2} \gamma_{s}^{2}-\frac{1}{2}\right) r^{2}+\frac{20}{63} g_{52}^{(3)}\left(\frac{2}{3} \gamma_{s}^{2}+\frac{1}{3}\right) r^{4}\right\} r
\end{array}\right.
$$

where $g_{12}^{(3)}, g_{32}^{(3)}$ and $g_{52}^{(3)}$ are the constants in the problem, corresponding to $\ell=1,3$ and 5 .

In the non-relativistic limit, we find that

$$
\left\{\begin{aligned}
\sqrt{2} F_{2}^{(3)} & =\sqrt{\frac{2}{7}} G_{12}^{(3)} r+\sqrt{\frac{2}{3}} G_{32}^{(3)} r^{3}+\sqrt{\frac{1}{21}} G_{52}^{(3)} r^{5} \\
\sqrt{2} F_{1}^{(3)} & =\frac{4}{\sqrt{35}} G_{12}^{(3)} r-\sqrt{\frac{1}{15}} G_{32}^{(3)} r^{3}-\sqrt{\frac{10}{21}} G_{52}^{(3)} r^{5} \\
F_{0}^{(3)} & =\frac{3}{\sqrt{35}} G_{12}^{(3)} r-\frac{2}{\sqrt{15}} G_{32}^{(3)} r^{3}+\sqrt{\frac{10}{21}} G_{52}^{(3)} r^{5}
\end{aligned}\right.
$$

Setting $\gamma_{s}=1$ in (17.14), we see that

$$
G_{12}^{(3)}=\sqrt{\frac{7}{3}} g_{12}^{(3)}, \quad G_{32}^{(3)}=-\frac{2}{5} g_{32}^{(3)}, \quad \text { and } \quad G_{52}^{(3)}=\frac{2}{3} \sqrt{\frac{2}{7}} g_{52}^{(3)}
$$

This can be obtained directly from (6.31). 


\section{Illustrative Examples II}

Here we confine ourselves to the cases in which both of the decay products have spins given by $s=1$ and $\sigma=1$. The corresponding decay amplitudes are worked out in Tables IIIa and IIIb. To further illustrate the techniques involved, $F^{J}$ 's are explicitly worked out with a few examples.

Table IIIa: Decay Amplitudes for $\ell-J=$ even $(s=1$ and $\sigma=1)$ $R$ stands for the rank.

\begin{tabular}{|c|c|c|c|c|}
\hline & $S$ & $R_{S}\left(n_{1}, n_{2}\right)$ & $R_{\ell}$ & $R_{J}$ \\
\hline 1 & 0 & $\dot{2}(0,0)$ & 0 & 0 \\
\hline 2 & 2 & $2(0,0)$ & 2 & 0 \\
\hline 3 & $0,1,2$ & $\dot{2}(0,0)$ & 1 & 1 \\
\hline 4 & $0,1,2$ & $2(1,0)$ & 1 & 1 \\
\hline 5 & $0,1,2$ & $2(0,1)$ & 1 & 1 \\
\hline 6 & 2 & $2(0,0)$ & 3 & 1 \\
\hline 7 & 2 & $2(1,1)$ & 0 & 2 \\
\hline 8 & $0,1,2$ & $\dot{2}(0,0)$ & 2 & 2 \\
\hline 9 & $0,1,2$ & $2(1,0)$ & 2 & 2 \\
\hline 10 & $0,1,2$ & $2(0,1)$ & 2 & 2 \\
\hline 11 & 2 & $2(0,0)$ & 4 & 2 \\
\hline 12 & 2 & $2(1,1)$ & 1 & 3 \\
\hline 13 & $0,1,2$ & $\dot{2}(0,0)$ & 3 & 3 \\
\hline
\end{tabular}

Table IIIa: Decay Amplitudes for $\ell-J=$ even $(s=1$ and $\sigma=1)$ $R$ stands for the rank.

\begin{tabular}{|c|c|c|c|c|c|}
\hline & $S$ & $R_{S}\left(n_{1}, n_{2}\right)$ & $R_{\ell}$ & & $R_{J}$ \\
\hline 14 & $0,1,2$ & $2(1,0)$ & 3 & & 3 \\
\hline 15 & $0,1,2$ & $2(0,1)$ & 3 & : & 3 \\
\hline 16 & 2 & $2(0,0)$ & $: 5$ & $\therefore$ & 3 \\
\hline 17 & 2 & $2(1,1)$ & 2 & . & 4 \\
\hline 18 & $0,1,2$ & $\dot{2}(0,0)$ & 4 & $::$ & 4 \\
\hline 19 & $0,1,2$ & $2(1,0)$ & 4 & $\therefore$ & 4 \\
\hline 20 & $0,1,2$ & $2(0,1)$ & 4 & $\therefore$ & 4 \\
\hline 21 & 2 & $2(0,0)$ & $: 6$ & $::$ & 4 \\
\hline 22 & 2 & $2(1,1)$ & 3 & $\therefore$ & 5 \\
\hline 23 & $0,1,2$ & $\dot{2}(0,0)$ & 5 & $::$ & 5 \\
\hline 24 & $0,1,2$ & $2(1,0)$ & 5 & $::$ & 5 \\
\hline 25 & $0,1,2$ & $2(0,1)$ & 5 & $::$ & 5 \\
\hline 26 & 2 & $2(0,0)$ & $: 7$ & $::$ & 5 \\
\hline
\end{tabular}


Table IIIb: Decay Amplitudes for $\ell-J=$ odd $(s=1$ and $\sigma=1)$

$R$ stands for the rank.

\begin{tabular}{|r|l|lll|}
\hline & $S$ & $R_{S}\left(n_{1}, n_{2}\right)$ & $R_{\ell}$ & $R_{J}$ \\
\hline 1 & 1 & $2\left(1^{\prime}, 1^{\prime}\right)$ & $1^{\prime}$ & 0 \\
\hline 2 & 1 & $2\left(1^{\prime}, 1^{\prime}\right)$ & 0 & $1^{\prime}$ \\
3 & 1,2 & $2\left(1^{\prime}, 0\right)$ & $\cdot 2^{\prime}$ & $1^{\prime}$ \\
4 & 1,2 & $2\left(0,1^{\prime}\right)$ & $\cdot 2^{\prime}$ & $1^{\prime}$ \\
5 & 1,2 & $2\left(1^{\prime}, 1^{\prime}\right)$ & $2^{\prime} \cdot 1$ \\
\hline 6 & 1,2 & $2\left(1^{\prime}, 1^{\prime}\right)$ & 1 & $2^{\prime}$ \\
7 & 1,2 & $2\left(1^{\prime}, 1\right)$ & $1^{\prime}$ & $2^{\prime}$ \\
8 & 1,2 & $2\left(1,1^{\prime}\right)$ & $1^{\prime}$ & $2^{\prime}$ \\
9 & 1,2 & $2\left(1^{\prime}, 1^{\prime}\right)$ & $3^{\prime}:$ & 2 \\
10 & 1,2 & $2\left(1^{\prime}, 0\right)$ & $\cdot 3^{\prime} \cdot 2^{\prime}$ \\
11 & 1,2 & $2\left(0,1^{\prime}\right)$ & $\cdot 3^{\prime} \cdot 2^{\prime}$ \\
\hline 12 & 1,2 & $2\left(1^{\prime}, 1^{\prime}\right)$ & 2 & $: 3^{\prime}$ \\
13 & 1,2 & $2\left(1^{\prime}, 1\right)$ & $2^{\prime} \cdot 3^{\prime}$ \\
14 & 1,2 & $2\left(1,1^{\prime}\right)$ & $2^{\prime} \cdot 3^{\prime}$ \\
15 & 1,2 & $2\left(1^{\prime}, 0\right)$ & $\cdot 4^{\prime}: 3^{\prime}$ \\
\hline
\end{tabular}

Table IIIb: Decay Amplitudes for $\ell-J=$ odd $(s=1$ and $\sigma=1)$ $R$ stands for the rank.

\begin{tabular}{|l|l|llll|}
\hline & $S$ & $R_{S}\left(n_{1}, n_{2}\right)$ & $R_{\ell}$ & $R_{J}$ \\
\hline 16 & 1,2 & $2\left(0,1^{\prime}\right)$ & $\cdot 4^{\prime}$ & $:$ & $3^{\prime}$ \\
17 & 1,2 & $2\left(1^{\prime}, 1^{\prime}\right)$ & $4^{\prime}$ & $:$ & 3 \\
\hline 18 & 1,2 & $2\left(1^{\prime}, 1^{\prime}\right)$ & 3 & $:$ & $4^{\prime}$ \\
19 & 1,2 & $2\left(1^{\prime}, 1\right)$ & $3^{\prime}$ & $:$ & $4^{\prime}$ \\
20 & 1,2 & $2\left(1,1^{\prime}\right)$ & $3^{\prime}$ & $:$ & $4^{\prime}$ \\
21 & 1,2 & $2\left(1^{\prime}, 1^{\prime}\right)$ & $5^{\prime}$ & $:$ & 4 \\
22 & 1,2 & $2\left(1^{\prime}, 0\right)$ & $\cdot 5^{\prime}$ & $:$ & $4^{\prime}$ \\
23 & 1,2 & $2\left(0,1^{\prime}\right)$ & $\cdot 5^{\prime}$ & $: \cdot$ & $4^{\prime}$ \\
\hline 24 & 1,2 & $2\left(1^{\prime}, 1^{\prime}\right)$ & 4 & $::$ & $5^{\prime}$ \\
25 & 1,2 & $2\left(1^{\prime}, 1\right)$ & $4^{\prime}$ & $: \cdot$ & $5^{\prime}$ \\
26 & 1,2 & $2\left(1,1^{\prime}\right)$ & $4^{\prime}$ & $: \cdot$ & $5^{\prime}$ \\
27 & 1,2 & $2\left(1^{\prime}, 0\right)$ & $\cdot 6^{\prime}$ & $::$ & $5^{\prime}$ \\
28 & 1,2 & $2\left(0,1^{\prime}\right)$ & $\cdot 6^{\prime}$ & $::$ & $5^{\prime}$ \\
29 & 1,2 & $2\left(1^{\prime}, 1^{\prime}\right)$ & $6^{\prime}$ & $::$ & 5 \\
& & & & \\
\hline
\end{tabular}




\section{$8.1 \quad \underline{0^{+} \rightarrow 1^{-}+1^{-}}$}

The allowed orbital angular momenta are $\ell=0$ and 2 (see rows 1 and 2, Table IIIa). Since $J=0$, we must have $S=\ell=0$ or 2 and $\lambda=\nu=0$ or 1 . So we find in the $J$ RF

$$
\begin{aligned}
A_{00}^{(0)}(s, \sigma ; 0) & =[\dot{\Psi}(0,0 ; 00)]_{w}=\tilde{g}_{\alpha \beta}(w) \Psi^{\alpha \beta}(0,0 ; 00) \\
& =\sum_{i} \Psi_{i i}(0,0 ; 00)=-\frac{1}{\sqrt{3}}\left(2+\gamma_{s} \gamma_{\sigma}\right) \\
A_{22}^{(0)}(s, \sigma ; 0) & =[\Psi(0,0 ; 20): \chi(20)]_{w} \\
& =\sum_{i j} \Psi_{i j}(0,0 ; 20) \chi_{j i}(20)=\frac{1}{3} \sqrt{\frac{2}{3}}\left(1+2 \gamma_{s} \gamma_{\sigma}\right)
\end{aligned}
$$

where we have used the notation $\dot{\psi}$ to indicate internal contraction of the rank- 2 tensor. The helicity-coupling amplitudes are, from (6.7),

$$
\left\{\begin{array}{l}
F_{ \pm \pm}^{(0)}=-g_{00}^{(0)}\left(\frac{1}{3} \gamma_{s} \gamma_{\sigma}+\frac{2}{3}\right)+\frac{1}{3} g_{22}^{(0)}\left(\frac{2}{3} \gamma_{s} \gamma_{\sigma}+\frac{1}{3}\right) r^{2} \\
F_{00}^{(0)}=g_{00}^{(0)}\left(\frac{1}{3} \gamma_{s} \gamma_{\sigma}+\frac{2}{3}\right)+\frac{2}{3} g_{22}^{(0)}\left(\frac{2}{3} \gamma_{s} \gamma_{\sigma}+\frac{1}{3}\right) r^{2}
\end{array}\right.
$$

We write the amplitudes so that the elements dependent on Lorentz factors are normalized to 1. Under the requirement of Bose symmetrization, the amplitudes above must be symmetric under interchange of $s$ and $\sigma$. We see that it is automatically satisfied.

Consider next the case of a very large mass for the parent particle. In the limit $w \rightarrow \infty$, we find

$$
\left\{\begin{array}{l}
F_{ \pm \pm}^{(0)} \rightarrow \frac{1}{3}\left(-g_{00}^{(0)}+\frac{2}{3} g_{22}^{(0)} r^{2}\right) \gamma_{s} \gamma_{\sigma} \rightarrow \frac{2}{9} g_{22}^{(0)} \gamma_{s} \gamma_{\sigma} r^{2} \\
F_{00}^{(0)} \rightarrow \frac{1}{3}\left(g_{00}^{(0)}+\frac{4}{3} g_{22}^{(0)} r^{2}\right) \gamma_{s} \gamma_{\sigma} \rightarrow \frac{4}{9} g_{22}^{(0)} \gamma_{s} \gamma_{\sigma} r^{2}
\end{array}\right.
$$

since $r^{2} \rightarrow \infty$ in this limit.

In the non-relativistic limit, the helicity-coupling amplitudes are given by

$$
\left\{\begin{aligned}
\sqrt{2} F_{ \pm \pm}^{J} & =\sqrt{\frac{2}{3}} G_{00}^{J}+\sqrt{\frac{1}{3}} G_{22}^{J} r^{2} \\
F_{00}^{J} & =-\sqrt{\frac{1}{3}} G_{00}^{J}+\sqrt{\frac{2}{3}} G_{22}^{J} r^{2}
\end{aligned}\right.
$$

with $J=0$. We set $\gamma_{s}=\gamma_{\sigma}=1$ and find

$$
G_{00}^{J}=-\sqrt{3} g_{00}^{J} \quad \text { and } \quad G_{22}^{J}=\sqrt{\frac{2}{3}} g_{22}^{J}
$$

This is consistent with the formula (6.28). 
$8.2 \stackrel{0^{-} \rightarrow 1^{-}+1^{-}}{ }$

The allowed orbital angular momentum is $\ell=1$ (see row 1 , Table IIIb). Since $J=0$, we must have $S=\ell=1$. The decay amplitude is

$$
A_{11}^{(0)}(s, \sigma ; 0)=\left[p, \Psi\left(1^{\prime}, 1^{\prime} ; 10\right), \chi\left(1^{\prime} 0\right)\right]_{w} r=\sqrt{2}(i w)
$$

and the corresponding helicity-coupling amplitude is

$$
F_{++}^{(0)}=(i w) g_{11}^{(0)} r, \quad F_{00}^{(0)}=0
$$

Again, the Bose symmetry is automatic. In the non-relativistic limit, we have $\sqrt{2} F_{++}^{(0)}=$ $-G_{11}^{(0)} r$ and $F_{00}^{(0)}=0$. So we obtain $G_{11}^{(0)}=-\sqrt{2}(i w) g_{11}^{(0)}$.

\section{$8.3 \stackrel{1^{-} \rightarrow 1^{+}+1^{-}}{ }$}

The allowed orbital angular momenta are $\ell=0$ and 2 (see rows 2, 3, 4 and 5, Table IIIb). Since $J=1$, we must have $S=1$ or 2 . The invariant amplitudes are, with $\delta=\lambda-\nu$,

$$
\begin{aligned}
& A_{0 S}^{(1)}(s, \sigma ; \delta)=\left[p, \Psi\left(1^{\prime}, 1^{\prime} ; S \delta\right), \phi^{*}\left(1^{\prime} \delta\right)\right]_{w}, \quad S=1 \\
& A_{2 S}^{(1)}(s, \sigma ; \delta)=\left[p, \Psi\left(1^{\prime}, 0 ; S \delta\right) \cdot \chi\left(2^{\prime} 0\right), \phi^{*}\left(1^{\prime} \delta\right)\right]_{w}, \quad S=1,2 \\
& B_{2 S}^{(1)}(s, \sigma ; \delta)=\left[p, \Psi\left(0,1^{\prime} ; S \delta\right) \cdot \chi\left(2^{\prime} 0\right), \phi^{*}\left(1^{\prime} \delta\right)\right]_{w}, \quad S=1,2 \\
& C_{2 S}^{(1)}(s, \sigma ; \delta)=\left[p, \Psi\left(1^{\prime}, 1^{\prime} ; S \delta\right), \chi\left(2^{\prime} 0\right) \cdot \phi^{*}(1 \delta)\right]_{w}, \quad S=1,2
\end{aligned}
$$

and so

$$
A_{01}^{(1)}(s, \sigma ;+)=\frac{1}{\sqrt{2}}(i w)\left(\gamma_{s}+\gamma_{\sigma}\right), \quad A_{01}^{(1)}(s, \sigma ; 0)=\sqrt{2}(i w)
$$

and

$$
\begin{aligned}
A_{21}^{(1)}(s, \sigma ;+) & =\frac{1}{3 \sqrt{2}}(i w)\left(2 \gamma_{\sigma}-\gamma_{s}\right), \quad B_{21}^{(1)}(s, \sigma ;+)=-\frac{1}{3 \sqrt{2}}(i w)\left(2 \gamma_{s}-\gamma_{\sigma}\right) \\
A_{21}^{(1)}(s, \sigma ; 0) & =-B_{21}^{(1)}(0)=-\frac{\sqrt{2}}{3}(i w) \\
C_{21}^{(1)}(s, \sigma ;+) & =-\frac{1}{3 \sqrt{2}}(i w)\left(\gamma_{s}+\gamma_{\sigma}\right), \quad C_{21}^{(1)}(s, \sigma ; 0)=\frac{2 \sqrt{2}}{3}(i w)
\end{aligned}
$$

We find, in addition,

$$
A_{22}^{(1)}(s, \sigma ;+)=\frac{(i w)}{3 \sqrt{2}}\left(2 \gamma_{\sigma}+\gamma_{s}\right), \quad A_{22}^{(1)}(s, \sigma ; 0)=0
$$


and

$$
\begin{array}{ll}
B_{22}^{(1)}(+)=\frac{(i w)}{3 \sqrt{2}}\left(2 \gamma_{s}+\gamma_{\sigma}\right), & B_{22}^{(1)}(0)=0 \\
C_{22}^{(1)}(+)=-\frac{(i w)}{3 \sqrt{2}}\left(\gamma_{\sigma}-\gamma_{s}\right), & C_{22}^{(1)}(0)=0
\end{array}
$$

So we define the most general helicity-coupling amplitudes to be

$$
F_{\lambda \nu}^{J}=F_{\lambda \nu}^{J}(A)+F_{\lambda \nu}^{J}(B)+F_{\lambda \nu}^{J}(C)
$$

We assign constants $g_{\ell S}^{J}$ 's, $f_{\ell S}^{J}$ 's and $h_{\ell S}^{J}$ 's to the amplitudes $A, B$ and $C$, respectively, and obtain

$$
\left\{\begin{aligned}
F_{++}^{(1)}= & (i w)\left[g_{01}^{(1)}-\frac{1}{3} g_{21}^{(1)} r^{2}+\frac{1}{3} f_{21}^{(1)} r^{2}+\frac{2}{3} h_{21}^{(1)} r^{2}\right] \\
F_{0+}^{(1)}= & (i w)\left[g_{01}^{(1)}\left(\frac{1}{2} \gamma_{\sigma}+\frac{1}{2} \gamma_{s}\right)-\frac{1}{3} h_{21}^{(1)}\left(\frac{1}{2} \gamma_{\sigma}+\frac{1}{2} \gamma_{s}\right) r^{2}\right. \\
& +\frac{1}{6} g_{21}^{(1)}\left(2 \gamma_{\sigma}-\gamma_{s}\right) r^{2}-\frac{1}{6} f_{21}^{(1)}\left(2 \gamma_{s}-\gamma_{\sigma}\right) r^{2}+\frac{1}{6} h_{22}^{(1)}\left(\gamma_{\sigma}-\gamma_{s}\right) r^{2} \\
& \left.-\frac{1}{2} g_{22}^{(1)}\left(\frac{2}{3} \gamma_{\sigma}+\frac{1}{3} \gamma_{s}\right) r^{2}-\frac{1}{2} f_{22}^{(1)}\left(\frac{2}{3} \gamma_{s}+\frac{1}{3} \gamma_{\sigma}\right) r^{2}\right] \\
F_{+0}^{(1)}= & (i w)\left[g_{01}^{(1)}\left(\frac{1}{2} \gamma_{\sigma}+\frac{1}{2} \gamma_{s}\right)-\frac{1}{3} h_{21}^{(1)}\left(\frac{1}{2} \gamma_{\sigma}+\frac{1}{2} \gamma_{s}\right) r^{2}\right. \\
& +\frac{1}{6} g_{21}^{(1)}\left(2 \gamma_{\sigma}-\gamma_{s}\right) r^{2}-\frac{1}{6} f_{21}^{(1)}\left(2 \gamma_{s}-\gamma_{\sigma}\right) r^{2}-\frac{1}{6} h_{22}^{(1)}\left(\gamma_{\sigma}-\gamma_{s}\right) r^{2} \\
& \left.+\frac{1}{2} g_{22}^{(1)}\left(\frac{2}{3} \gamma_{\sigma}+\frac{1}{3} \gamma_{s}\right) r^{2}+\frac{1}{2} f_{22}^{(1)}\left(\frac{2}{3} \gamma_{s}+\frac{1}{3} \gamma_{\sigma}\right) r^{2}\right]
\end{aligned}\right.
$$

We conclude that the three helicity-coupling amplitudes $F$ depend on a total of seven parameters, three $g$ 's, two $f$ 's and two $h$ 's. The terms with $h_{22}^{(1)}$ are purely relativistic; they vanish in the limit $\gamma_{s}=\gamma_{\sigma}=1$.

This example can be used to also evaluate $1^{+} \rightarrow 1^{-}+1^{-}$with the two vector states being identical. The resulting helicity-coupling amplitudes are, from (6.17),

$$
\left\{\begin{array}{l}
F_{++}^{(1)}=0 \\
F_{0+}^{(1)}=(i w)\left[\frac{1}{4}\left(g_{21}^{(1)}-f_{21}^{(1)}\right)\left(\gamma_{\sigma}-\gamma_{s}\right)-\frac{1}{2}\left(g_{22}^{(1)}-f_{22}^{(1)}\right)\left(\frac{1}{2} \gamma_{\sigma}+\frac{1}{2} \gamma_{s}\right)\right] r^{2} \\
F_{+0}^{(1)}=(i w)\left[\frac{1}{4}\left(g_{21}^{(1)}-f_{21}^{(1)}\right)\left(\gamma_{\sigma}-\gamma_{s}\right)+\frac{1}{2}\left(g_{22}^{(1)}-f_{22}^{(1)}\right)\left(\frac{1}{2} \gamma_{\sigma}+\frac{1}{2} \gamma_{s}\right)\right] r^{2}
\end{array}\right.
$$

and the amplitudes depend only on two parameters $\left(g_{21}^{(1)}-f_{21}^{(1)}\right)$ and $\left(g_{22}^{(1)}-f_{22}^{(1)}\right)$. The first term is purely relativistic and so it vanishes in the limit $\gamma_{s}=\gamma_{\sigma}=1$. In addition, one may 
expect that the first term should remain small and insignificant, since $\gamma_{s}$ should be nearly equal to $\gamma_{\sigma}$ independent of the size of $w$ compared to $m$ or $\mu$.

The helicity-coupling amplitudes are, in the non-relativistic limit,

$$
\left\{\begin{array}{l}
\sqrt{2} F_{++}^{(1)}=\frac{1}{\sqrt{3}} G_{01}^{(1)}-\sqrt{\frac{2}{3}} G_{21}^{(1)} r^{2} \\
\sqrt{2} F_{0+}^{(1)}=\frac{1}{\sqrt{3}} G_{01}^{(1)}+\frac{1}{\sqrt{6}} G_{21}^{(1)} r^{2}-\frac{1}{\sqrt{2}} G_{22}^{(1)} r^{2} \\
\sqrt{2} F_{+0}^{(1)}=\frac{1}{\sqrt{3}} G_{01}^{(1)}+\frac{1}{\sqrt{6}} G_{21}^{(1)} r^{2}+\frac{1}{\sqrt{2}} G_{22}^{(1)} r^{2}
\end{array}\right.
$$

So there are just three parameters in this limit. Note that the summations indicated by $\left(\underline{6.3 a}\right.$ ) and $(\underline{6.3 b})$ hold for the formula above. By setting $\gamma_{s}=\gamma_{\sigma}=1$, we obtain

$$
\left\{\begin{array}{l}
G_{01}^{(1)}=\sqrt{6}(i w) g_{01}^{(1)}, \\
G_{22}^{(1)}=(i w)\left(g_{22}^{(1)}+f_{22}^{(1)}\right)
\end{array} \quad G_{21}^{(1)}=\frac{1}{\sqrt{3}}(i w)\left(g_{21}^{(1)}-f_{21}^{(1)}-2 h_{21}^{(1)}\right)\right.
$$

Or, alternatively, we could have used (6.28) to obtain the same result.

\section{$8.4 \quad \underline{1^{+} \rightarrow 1^{+}+1^{-}}$}

The allowed orbital angular momenta are $\ell=1$ and 3 (see rows 3, 4, 5 and 6, Table IIIa). Since $J=1$, we can have $S=0,1$ or 2 . The invariant amplitudes are, with $\delta=\lambda-\nu$,

$$
\left\{\begin{array}{l}
A_{1 S}^{(1)}(s, \sigma ; \delta)=\left[\Psi(1,0 ; S \delta) \cdot \chi(10) \phi^{*}(1 \delta)\right]_{w}, \quad S=0,1,2 \\
B_{1 S}^{(1)}(s, \sigma ; \delta)=\left[\Psi(0,1 ; S \delta) \cdot \chi(10) \phi^{*}(1 \delta)\right]_{w}, \quad S=0,1,2 \\
C_{1 S}^{(1)}(s, \sigma ; \delta)=\left[\dot{\Psi}(0,0 ; S \delta) \chi(10) \cdot \phi^{*}(1 \delta)\right]_{w}, \quad S=0,1,2 \\
A_{3 S}^{(1)}(s, \sigma ; \delta)=\left[\Psi(0,0 ; S \delta): \chi(30) \cdot \phi^{*}(1 \delta)\right]_{w}, \quad S=2
\end{array}\right.
$$

We find

$$
\begin{aligned}
& A_{10}^{(1)}(s, \sigma ; 0)=-\frac{1}{\sqrt{3}} \gamma_{s} \gamma_{\sigma}, \quad B_{10}^{(1)}(0)=-\frac{1}{\sqrt{3}} \gamma_{s} \gamma_{\sigma} \quad C_{10}^{(1)}(0)=-\frac{1}{\sqrt{3}}\left(2+\gamma_{s} \gamma_{\sigma}\right) \\
& A_{11}^{(1)}(s, \sigma ; 0)=0, \quad B_{11}^{(1)}(0)=0, \quad C_{11}^{(1)}(0)=0 \\
& A_{11}^{(1)}(s, \sigma ;+)=-\frac{1}{\sqrt{2}} \gamma_{s}, \quad B_{11}^{(1)}(s, \sigma ;+)=+\frac{1}{\sqrt{2}} \gamma_{\sigma} \quad C_{11}^{(1)}(+)=0 \\
& A_{12}^{(1)}(s, \sigma ; 0)=\sqrt{\frac{2}{3}} \gamma_{s} \gamma_{\sigma}, \quad B_{12}^{(1)}(s, \sigma ; 0)=\sqrt{\frac{2}{3}} \gamma_{s} \gamma_{\sigma} \quad C_{12}^{(1)}(s, \sigma ; 0)=-\sqrt{\frac{2}{3}}\left(1-\gamma_{s} \gamma_{\sigma}\right) \\
& A_{12}^{(1)}(s, \sigma ;+)=\frac{1}{\sqrt{2}} \gamma_{s}, \quad B_{12}^{(1)}(s, \sigma ;+)=\frac{1}{\sqrt{2}} \gamma_{\sigma} r, \quad C_{12}^{(1)}(+)=0
\end{aligned}
$$


and

$$
A_{32}^{(1)}(s, \sigma ; 0)=\frac{1}{5} \sqrt{\frac{2}{3}}\left(1+2 \gamma_{s} \gamma_{\sigma}\right), \quad A_{32}^{(1)}(s, \sigma ;+)=-\frac{1}{5 \sqrt{2}}\left(\gamma_{s}+\gamma_{\sigma}\right)
$$

We assign constants $g_{\ell S}^{J}$ 's, $f_{\ell S}^{J}$ 's and $h_{\ell S}^{J}$ 's to the amplitudes $A, B$ and $C$, respectively, and obtain

$$
\left\{\begin{aligned}
& F_{++}^{(1)}=-\frac{1}{3} {\left[\left(g_{10}^{(1)}+f_{10}^{(1)}\right) \gamma_{s} \gamma_{\sigma}+3 h_{10}^{(1)}\left(\frac{2}{3}+\frac{1}{3} \gamma_{s} \gamma_{\sigma}\right)\right] r } \\
& \quad+\frac{1}{3}\left[\left(g_{12}^{(1)}+f_{12}^{(1)}\right) \gamma_{s} \gamma_{\sigma}+h_{12}^{(1)}\left(1-\gamma_{s} \gamma_{\sigma}\right)\right] r+\frac{1}{5} g_{32}^{(1)}\left(\frac{1}{3}+\frac{2}{3} \gamma_{s} \gamma_{\sigma}\right) r^{3} \\
& F_{0+}^{(1)}= \frac{1}{2}\left(g_{11}^{(1)} \gamma_{s}-f_{11}^{(1)} \gamma_{\sigma}\right) r+\frac{1}{2}\left(g_{12}^{(1)} \gamma_{s}+f_{12}^{(1)} \gamma_{\sigma}\right) r-\frac{1}{5} g_{32}^{(1)}\left(\frac{1}{2} \gamma_{s}+\frac{1}{2} \gamma_{\sigma}\right) r^{3} \\
& F_{+0}^{(1)}=-\frac{1}{2}\left(g_{11}^{(1)} \gamma_{s}-f_{11}^{(1)} \gamma_{\sigma}\right) r+\frac{1}{2}\left(g_{12}^{(1)} \gamma_{s}+f_{12}^{(1)} \gamma_{\sigma}\right) r-\frac{1}{5} g_{32}^{(1)}\left(\frac{1}{2} \gamma_{s}+\frac{1}{2} \gamma_{\sigma}\right) r^{3} \\
& F_{00}^{(1)}=\frac{1}{3}\left[\left(g_{10}^{(1)}+f_{10}^{(1)}\right) \gamma_{s} \gamma_{\sigma}+h_{10}^{(1)}\left(2+\gamma_{s} \gamma_{\sigma}\right)\right] r \\
& \quad+\frac{2}{3}\left[\left(g_{12}^{(1)}+f_{12}^{(1)}\right) \gamma_{s} \gamma_{\sigma}+h_{12}^{(1)}\left(1-\gamma_{s} \gamma_{\sigma}\right)\right] r+\frac{2}{5} g_{32}^{(1)}\left(\frac{1}{3}+\frac{2}{3} \gamma_{s} \gamma_{\sigma}\right) r^{3}
\end{aligned}\right.
$$

So the amplitudes depend on eight parameters; $\left(g_{10}^{(1)}+f_{10}^{(1)}\right), g_{11}^{(1)}, g_{12}^{(1)}, g_{32}^{(1)}, f_{11}^{(1)}, f_{12}^{(1)}, h_{10}^{(1)}$ and $h_{12}^{(1)}$. It should be noted that the term proportional to $h_{12}^{(1)}$ is purely relativistic, since $\left(1-\gamma_{s} \gamma_{\sigma}\right)$ vanishes in the non-relativistic limit $\gamma_{s}=\gamma_{\sigma}=1$.

This example can also be applied to the case $1^{-} \rightarrow 1^{-}+1^{-}$, where the two vector states are identical. We need to carry out the operation given in (6.17). The resulting amplitudes are

$$
\left\{\begin{array}{l}
F_{++}^{(1)}=0, \quad F_{0+}^{(1)}=\frac{1}{2}\left(g_{11}^{(1)}-f_{11}^{(1)}\right)\left(\frac{1}{2} \gamma_{s}+\frac{1}{2} \gamma_{\sigma}\right) r+\frac{1}{4}\left(g_{12}^{(1)}-f_{12}^{(1)}\right)\left(\gamma_{s}-\gamma_{\sigma}\right) r \\
F_{00}^{(1)}=0, \quad F_{+0}^{(1)}=-\frac{1}{2}\left(g_{11}^{(1)}-f_{11}^{(1)}\right)\left(\frac{1}{2} \gamma_{s}+\frac{1}{2} \gamma_{\sigma}\right) r+\frac{1}{4}\left(g_{12}^{(1)}-f_{12}^{(1)}\right)\left(\gamma_{s}-\gamma_{\sigma}\right) r
\end{array}\right.
$$

The amplitudes depend on two parameters $\left(g_{11}^{(1)}-f_{11}^{(1)}\right)$ and $\left(g_{12}^{(1)}-f_{12}^{(1)}\right)$. The terms corresponding to the second parameter, with $\ell+S=$ odd, vanish in the non-relativistic limit $\gamma_{s}=\gamma_{\sigma}=1$. 
The helicity-coupling amplitudes are, in the non-relativistic limit,

$$
\left\{\begin{aligned}
\sqrt{2} F_{++}^{(1)} & =\sqrt{\frac{2}{3}} G_{10}^{(1)} r-\sqrt{\frac{2}{15}} G_{12}^{(1)} r+\frac{1}{\sqrt{5}} G_{32}^{(1)} r^{3} \\
\sqrt{2} F_{0+}^{(1)} & =\frac{1}{\sqrt{2}} G_{11}^{(1)} r-\sqrt{\frac{3}{10}} G_{12}^{(1)} r-\frac{1}{\sqrt{5}} G_{32}^{(1)} r^{3} \\
\sqrt{2} F_{+0}^{(1)} & =-\frac{1}{\sqrt{2}} G_{11}^{(1)} r-\sqrt{\frac{3}{10}} G_{12}^{(1)} r-\frac{1}{\sqrt{5}} G_{32}^{(1)} r^{3} \\
F_{00}^{(1)} & =-\frac{1}{\sqrt{3}} G_{10}^{(1)} r-\frac{2}{\sqrt{15}} G_{12}^{(1)} r+\sqrt{\frac{2}{5}} G_{32}^{(1)} r^{3}
\end{aligned}\right.
$$

In this case, there are four independent parameters, $G_{10}^{(1)}, G_{11}^{(1)}, G_{12}^{(1)}, G_{32}^{(1)}$. It is instructive to note that the normalization conditions given by $(6.3 \mathrm{~b})$ and $(6.3 \mathrm{~b})$ are satisfied by the formula above. Once again, we set $\gamma_{s}=\gamma_{\sigma}=1$ in (8.21) and obtain

$$
\begin{cases}G_{10}^{(1)}=-\frac{1}{\sqrt{3}}\left(g_{10}^{(1)}+f_{10}^{(1)}+3 h_{10}^{(1)}\right), & G_{11}^{(1)}=g_{11}^{(1)}-f_{11}^{(1)} \\ G_{12}^{(1)}=-\sqrt{\frac{5}{3}}\left(g_{12}^{(1)}+f_{12}^{(1)}\right), & G_{32}^{(1)}=\sqrt{\frac{2}{5}} g_{32}^{(1)}\end{cases}
$$

which is consistent with (6.28).

\section{Illustrative Examples III}

We continue illustrating the decay amplitudes by considering the decay modes with $J=$ $s=\sigma=2$. In order to make our paper concise and readable, we merely show the allowed amplitudes in tabular forms and exhibit a few explicit examples of a given $J, \ell$ and $S$ but we do not work out $F_{\lambda_{s} \lambda_{\sigma}}^{J}$; the reader is invited to consult our $\mathrm{C}++$ program posted online (see Section 10) for the full details.

The decay amplitudes with $s+\sigma+\ell_{J}=$ even (odd) are given in Table IVa (Table IVb). 
Table IVa. Decay Amplitudes for $2 \rightarrow 2+2$

$R$ stands for the rank.

\begin{tabular}{|c|c|c|c|c|c|}
\hline & $S$ & $R\left(n_{1}, n_{2}\right)$ & & $R_{\ell}$ & $R_{J}$ \\
\hline 1 & 2 & $\dot{4}(1,1)$ & & 0 & 2 \\
\hline 2 & $0-4$ & $\ddot{4}(0,0)$ & & 2 & 2 \\
\hline 3 & $0-4$ & $\dot{4}(1,0)$ & . & 2 & 2 \\
\hline 4 & $0-4$ & $\dot{4}(0,1)$ & . & 2 & 2 \\
\hline 5 & $0-4$ & $4(2,0)$ & : & 2 & 2 \\
\hline 6 & $0-4$ & $4(1,1)$ & : & 2 & 2 \\
\hline 7 & $0-4$ & $4(0,2)$ & : & 2 & 2 \\
\hline 8 & $2,3,4$ & $\dot{4}(0,0)$ & : & 4 & 2 \\
\hline 9 & $2,3,4$ & $4(1,0)$ & $\therefore$ & 4 & 2 \\
\hline 10 & $2,3,4$ & $4(0,1)$ & $\therefore$ & 4 & 2 \\
\hline 11 & 4 & $4(0,0)$ & $::$ & 6 & 2 \\
\hline
\end{tabular}

The decay amplitudes are, in the $J \mathrm{RF}$,

Row 1, Table IVa:

$$
A_{0 S}^{(2)}(s, \sigma ; \delta)=\Psi_{\nu \tau}^{\alpha \nu}(1,1 ; S \delta) \tilde{g}^{\tau \rho}(w) \phi_{\alpha \rho}^{*}(2 \delta)
$$

Row 7, Table IVa:

$$
A_{2 S}^{(2)}(s, \sigma ; \delta)=\Psi^{\alpha \beta \mu \nu}(0,2 ; S \delta) \chi_{\alpha \beta}(20) \phi_{\mu \nu}^{*}(2 \delta)
$$

Row 9:, Table IVa:

$$
A_{4 S}^{(2)}(s, \sigma ; \delta)=\Psi^{\alpha \beta \mu \nu}(1,0 ; S \delta) \chi_{\beta \mu \nu \tau}(40) \tilde{g}^{\tau \rho}(w) \phi_{\rho \alpha}^{*}(2 \delta)
$$


Table IVb. Decay Amplitudes for $2 \rightarrow 2+2$

$R$ stands for the rank.

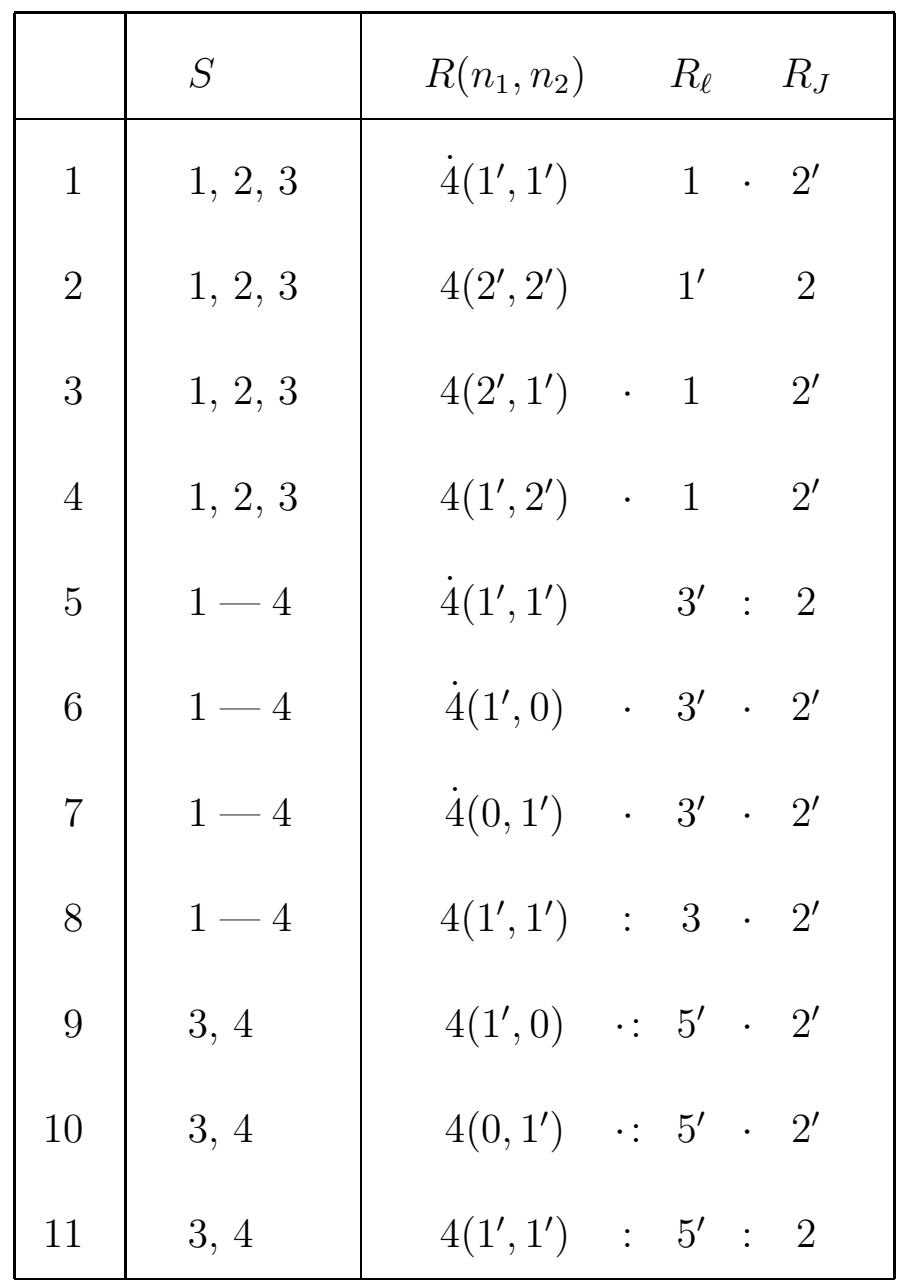

and

Row 1, Table IVb:

$$
A_{1 S}^{(2)}(s, \sigma ; \delta)=\epsilon_{\alpha \beta \mu \nu} p^{\alpha} \Psi^{\beta \tau \rho \mu}\left(1^{\prime}, 1^{\prime} ; S \delta\right) \tilde{g}_{\tau \rho}(w) \chi_{\gamma}(10) \phi^{* \gamma \nu}\left(2^{\prime} \delta\right)
$$

Row 2, Table IVb:

$$
A_{1 S}^{(2)}(s, \sigma ; \delta)=\epsilon_{\alpha \beta \mu \nu} p^{\alpha} \Psi^{\beta \tau \rho \mu}\left(2^{\prime}, 2^{\prime} ; S \delta\right) \chi^{\nu}\left(1^{\prime} 0\right) \phi_{\tau \rho}^{*}(2 \delta)
$$

Row 8, Table IVb:

$$
A_{3 S}^{(2)}(s, \sigma ; \delta)=\epsilon_{\alpha \beta \mu \nu} p^{\alpha} \Psi^{\beta \tau \rho \mu}\left(1^{\prime}, 1^{\prime} ; S \delta\right) \chi_{\tau \rho \gamma}(30) \phi^{* \gamma \nu}\left(2^{\prime} \delta\right)
$$




\section{Amplitudes for Sequential Decay}

We now turn to a discussion of the decay amplitudes for which the daughter states $s$ and $\sigma$ decay into, e.g. two or more pions. To illustrate the techniques necessary for such processes, it is sufficient to consider the decay $s \rightarrow \pi \pi$ and $\sigma \rightarrow 3 \pi$. So $s$ can represent any one of $f_{0}(600), \rho(770)$ or $f_{2}(1275)$, while $\sigma$ could be $\omega(782), a_{1}(1260), a_{2}(1320)$ or $\pi_{2}(1670)$. The "analyzer" for the $\pi \pi$ system is the momentum of one of the pions in its appropriate rest frame, whereas the analyzer for the $3 \pi$ system has been chosen, for the purpose of illustration in this section, as the normal to the decay plane in its rest frame.

Consider the decay amplitude $J \rightarrow s+\sigma$ in the $J \mathrm{RF}$ as given in (6.1). Let $(\vec{x}, \vec{y}, \vec{z})$ stand for the coordinate system in the $J \mathrm{RF}$, in which the angles $(\theta, \phi)$ describe the orientation of $s$. We go into the $s \mathrm{RF}$ and choose the coordinate axes $\left(\vec{x}_{s}, \vec{y}_{s}, \vec{z}_{s}\right)$ such that

$$
\left.\vec{z}_{s} \propto \vec{s}\right|_{J \mathrm{RF}}, \quad \vec{y}_{s} \propto \vec{z} \times\left.\vec{s}\right|_{J \mathrm{RF}} \quad \text { and } \quad \vec{x}_{s}=\vec{y}_{s} \times\left.\vec{z}_{s}\right|_{s \mathrm{RF}}
$$

Here we have taken advantage of the fact that the direction of $s$ in the $J$ RF is preserved in the $s \mathrm{RF}$, and the vector $\vec{z} \times \vec{s}$ is normal to the direction of the Lorentz transformation and hence invariant under the transformation. We note that this particular choice of the coordinate system in the $s \mathrm{RF}$ is dictated by the choice of the arguments $(\phi, \theta, 0)$ of the $D$-function which appears in (6.1) 3. Let $\left(\theta_{s}, \phi_{s}\right)$ describe the orientation of one of the pions for the decay $s \rightarrow \pi \pi$ in the $s \mathrm{RF}$. Then, the decay amplitude is

$$
\mathcal{M}_{\lambda}^{s}\left(\theta_{s}, \phi_{s}\right)=\sqrt{\frac{2 s+1}{4 \pi}} F^{s} D_{\lambda 0}^{s *}\left(\phi_{s}, \theta_{s}, 0\right)
$$

where $F^{s}$ is the complex decay-coupling constant, which could be absorbed into $F_{\lambda \nu}^{J}$. The description of the decay $\sigma \rightarrow 3 \pi$ proceeds along a similar path. The coordinate axes $\left(\vec{x}_{\sigma}, \vec{y}_{\sigma}, \vec{z}_{\sigma}\right)$ in the $\sigma \mathrm{RF}$ are

$$
\left.\vec{z}_{\sigma} \propto \vec{\sigma}\right|_{J \mathrm{RF}}=-\left.\vec{s}\right|_{J \mathrm{RF}}, \quad \vec{y}_{\sigma} \propto \vec{z} \times\left.\vec{\sigma}\right|_{J \mathrm{RF}}=-\vec{z} \times\left.\vec{s}\right|_{J \mathrm{RF}} \quad \text { and } \quad \vec{x}_{\sigma}=\vec{y}_{\sigma} \times\left.\vec{z}_{\sigma}\right|_{\sigma \mathrm{RF}}
$$

Let $\left(\theta_{\sigma}, \phi_{\sigma}\right)$ describe the orientation of the normal to the decay plane for $\sigma \rightarrow 3 \pi$ in the $\sigma \mathrm{RF}$, and let $\varphi_{\sigma}$ fix the direction of one of the pions in the decay plane. Then, the decay amplitude is 3 ]

$$
\mathcal{M}_{\nu}^{\sigma}\left(\theta_{\sigma}, \phi_{\sigma}, \varphi_{\sigma}\right)=\sqrt{\frac{2 \sigma+1}{8 \pi^{2}}} \sum_{\zeta} F_{\zeta}^{\sigma} D_{\nu \zeta}^{\sigma *}\left(\phi_{\sigma}, \theta_{\sigma}, \varphi_{\sigma}\right)
$$


where $\zeta$ is the spin projection of $\sigma$ along the decay normal, i.e. $-\sigma \leq \zeta \leq+\sigma$, and hence it is a rotationally invariant quantum number. $F_{\zeta}^{\sigma}$ is the complex decay-coupling constant, which satisfies, from parity conservation in the decay,

$$
F_{\zeta}^{\sigma}=\eta_{\sigma}(-)^{\zeta+1} F_{\zeta}^{\sigma}
$$

So, if $\eta_{\sigma}=+1(-1)$, we must have $F_{\zeta}^{\sigma}=0$ for $\zeta=$ even (odd). Consider, for example, that $\sigma$ is the $\omega(782)$ and we examine its decay into $\pi^{+} \pi^{0} \pi^{-}$. Since $F_{ \pm}^{\sigma}=0$, the only nonzero decaycoupling constant is $F_{0}^{\sigma}$, which implies that the $D$-function with $\zeta=0$ is independent of $\varphi_{\sigma}$. Integrate the resulting angular distribution over the angle; this is equivalent to multiplying the decay amplitude (10.4) by $\sqrt{2 \pi}$ and setting $\varphi_{\sigma}=0$. We see that the amplitude is exactly the same as (10.2) for $\rho \rightarrow \pi \pi$, a well-known result.

We are now ready to write down the full decay amplitude for $J \rightarrow s+\sigma, s \rightarrow \pi \pi$ and $\sigma \rightarrow 3 \pi$. It is simply the product of (6.1), (10.2) and (10.4), i.e.

$$
\mathcal{M}_{\lambda \nu}^{J}(M ; \theta, \phi) \mathcal{M}_{\lambda}^{s}\left(\theta_{s}, \phi_{s}\right) \mathcal{M}_{\nu}^{\sigma}\left(\theta_{\sigma}, \phi_{\sigma}, \varphi_{\sigma}\right)
$$

where all the rotationally invariant quantum numbers appear either as super- or sub-scripts. The overall amplitude depends on seven angles and on $M$ as arguments. Here the spin projection $M$ of $J$ in the $J$ RF appears an argument in $\mathcal{M}$, since it is not a rotationally invariant quantum number. We note that the set of angles $\left\{\theta_{\sigma}, \phi_{\sigma}, \varphi_{\sigma}\right\}$ are in reality the familiar Euler angles which fix the orientation of the $3 \pi$ system in the $\sigma \mathrm{RF}$.

\section{Conclusions and Discussions}

We have given in this paper a general prescription for incorporating the functional dependence of the Lorentz factors in a fully relativistic two-body decay amplitude. We believe that our approach given in this paper is a proper and a natural way of combining the helicity formalism of Jacob and Wick[8] with the tensor wave functions of Rarita and Schwinger [9], Behrends and Fronsdal[10] and Zemach[11]. The central idea for such an approach has been

presented in Section 6, and a number of examples of the decay $J \rightarrow s+\sigma$ have been given in Section 7, 8 and 9. 
We need to elaborate on the measurability of the Lorentz factors worked out in this paper. For the purpose, we first point out that, for application to partial-wave analyses, the amplitudes $A_{\ell S}^{J}$ must include not only the Breit-Wigner forms for the daughter states $s$ and $\sigma$ but also those for parent states $J$ (for the so-called mass-independent global fits to the experimental data). It is best to illustrate the proposed formalism with two examples for the decay process:

\begin{tabular}{|c|cc|c|c|}
\hline$J \rightarrow s+\sigma$ & $w_{0}(\mathrm{MeV})$ & $\Gamma_{0}(\mathrm{MeV})$ & $\mathrm{r}(\mathrm{MeV} / c)$ & Amplitudes \\
\hline$\pi_{2}(1670) \rightarrow \rho+\pi$ & 1672.4 & 259 & $2 \times 648$ & Section 7.2 \\
$f_{1}(1285) \rightarrow \rho+\rho$ & 1281.8 & 24.1 & & Section 8.4 \\
\hline
\end{tabular}

Consider first the decay of the $\pi_{2}(1670)$. The relative momentum $r \simeq 1.30 \mathrm{GeV}$ is large enough for $\gamma_{s}$ to be substantially different from one; it is a variable which depend on the $\rho \pi$ effective mass $w$, as it ranges over, e.g. from $w_{0}-\Gamma_{0}$ to $w_{0}+\Gamma_{0}$ and, in addition, it depends on the $\pi \pi$ effective mass $m_{12}$ from the decay $\rho \rightarrow \pi+\pi$, designating the pions from the $\rho$ decay by the subscripts 1 and 2 . The Lorentz factor is, in the $J$ RF,

$$
\gamma_{s}=\frac{\sqrt{q^{2}+m_{12}^{2}}}{m_{12}}
$$

where the effective mass $m_{12}$ is subject to the Breit-Wigner form for the $\rho$. So the coefficient of $\gamma_{s}$ should be measurable, and hence add important new information on the decay property. We advocate that both approaches, non-relativistic $[([6.2)]$ and relativistic $[([6.7)]$, be used on the data and assay the difference by comparing the fitted parameters using (6.31). Here the comparison should be carried out with the amplitudes at their maxima, i.e. $w=w_{\text {th }}$ is replaced by $w=w_{0}$ where $w_{0}$ is the mass of the $\pi_{2}(1670)$, and $m_{12}$ of (11.1) by $m_{\rho}$, the mass of the $\rho$.

The situation with the second example above is different. We note that $w_{0}=1281.8$ $\mathrm{MeV}$ is less than $2 w_{\rho}, \mathrm{b}$ Let the first $\rho(s)$ decay into pions 1 and 2 , while the second $\rho(\sigma)$ into pions 3 and 4 . We need to set, in the $J$ RF,

$$
\gamma_{s}=\frac{\sqrt{q^{2}+m_{12}^{2}}}{m_{12}}, \quad \gamma_{\sigma}=\frac{\sqrt{q^{2}+m_{34}^{2}}}{m_{34}}
$$

where $m_{12}$ and $m_{34}$ stand for the appropriate two-body effective masses. They are constrained by the two Breit-Wigner forms for the $\rho$. Because of the narrow width of the

b Another example with a similar situation is the putative decay $\omega \rightarrow \rho+\pi$. 
$f_{1}(1285)$, the $w$-mass dependence of the amplitudes is absent, i.e. for all practical purposes, we could simply set $w=w_{0}$, where $w_{0}$ is the mass of the $f_{1}(1285)$. The functional dependence of $\gamma_{s}$ and $\gamma_{\sigma}$ is important, owing to the finite width of the $\rho$, and it should be included in the analysis. Once again, both approaches, non-relativistic [(6.2)] and relativistic [([6.7)], should be used in the analysis of data. The resulting parameters can be compared using the formula (6.28), where the first requirement of $w=w_{\text {th }}$ is replaced by $w=w_{0}$. Here the second requirement $\gamma_{s}=\gamma_{\sigma}=1$ remains valid, since the Lorentz factors (11.2) should be set at $m_{12}=m_{34}=w_{0} / 2($ so that $q=0)$.

A comment on the example of Section 8.1 is in order. Consider the hypothetical Higgs decay $H \rightarrow W^{+}+W^{-}$. As the Higgs mass increases to infinity, we see that the helicitycoupling amplitudes $F_{ \pm \pm}^{(0)}$ and $F_{00}^{(0)}$ become equally large [see (8.3)], indicating that the vector character of the $W$ bosons is preserved in this limit. This is in contrast to the well-known equivalence theorem[12], which states that, in the infinite mass limit of the Higgs particle, the $W$ bosons behave like scalars (the Goldstone bosons). This phenomenon can be traced to the interactions of the $W$ bosons with the scalar field, with the result that the final $W$ bosons contain both spin-1 and spin-0 components in the Standard Model. It is clear from this point of view that our helicity-coupling amplitudes have nothing to do with the interacting $W$ bosons, and hence our phenomenological model does not apply to the hypothetical Higgs decay $H \rightarrow W^{+}+W^{-}$in the large-mass limit.

The Zemach amplitudes[11] are commonly used used in partial-wave analyses. They correspond to the non-relativistic limit, i.e. $\gamma_{s}=1$ and $\gamma_{\sigma}=1$, of the amplitudes worked out in this paper. However, we need to caution the reader that we do not imply that the Zemach amplitudes are correct only in the non-relativistic limit. We merely wish to emphasize that our decay amplitudes with the Lorentz factors are an equally valid approach to writing down the amplitudes; our approach is more general, only in the sense that our decay amplitudes lead to the Zemach amplitudes when we set $\gamma_{s}=1$ and $\gamma_{\sigma}=1$.

In our formulation of the decay amplitudes, the orbital angular momentum $\ell$ leads naturally to the barrier factor $r^{\ell}$ in the formula. Since the factor is necessary to ensure that the amplitudes are singularity-free at the threshold, i.e. $r \rightarrow 0$, we need to "damp" it in the limit $r \rightarrow \infty$. This is accomplished by substituting $r^{\ell}$ with the Blatt-Weisskopf (BW) barrier factors [13]. The practitioners of partial-wave analyses in fact use the BW factors in their 
Zemach amplitudes. Let $f_{\ell}(u)$ be the BW factor where $u=r / r_{0}$ and $r_{0}=0.1973 \mathrm{GeV} / c$. Here $r_{0}$ corresponds to a sphere of radius 1 fermi for the strong interaction responsible for the decay in the coordinate space. It can be shown that

$$
\left.f_{\ell}(u)\right|_{u \rightarrow 0}=\frac{2^{\ell} \ell !}{(2 \ell) !} u^{\ell},\left.\quad f_{\ell}(u)\right|_{u \rightarrow \infty}=1
$$

which show that $f_{\ell}(u)$ is indeed the BW factor with the appropriate expected properties. We give below the explicit expressions of the BW factor for $\ell=0 \rightarrow 5$ :

$$
\begin{aligned}
& f_{0}(u)=1, \quad f_{1}(u)=u\left[1+u^{2}\right]^{-1 / 2}, \quad f_{2}(u)=u^{2}\left[\left(u^{2}-3\right)^{2}+9 u^{2}\right]^{-1 / 2}, \\
& f_{3}(u)=u^{3}\left[9\left(2 u^{2}-5\right)^{2}+u^{2}\left(u^{2}-15\right)^{2}\right]^{-1 / 2} \\
& f_{4}(u)=u^{4}\left[\left(u^{4}-45 u^{2}+105\right)^{2}+25 u^{2}\left(2 u^{2}-21\right)^{2}\right]^{-1 / 2} \\
& f_{5}(u)=u^{5}\left[225\left(u^{4}-28 u^{2}+63\right)^{2}+u^{2}\left(u^{4}-105 u^{2}+945\right)^{2}\right]^{-1 / 2}
\end{aligned}
$$

The helicity-coupling amplitude $F$ as given in (6.7) involves a factor $A_{\ell S}^{J}(s, \sigma ; \delta) r^{\ell}$ which must now be replaced by

$$
A_{\ell S}^{J}(s, \sigma ; \delta) \rightarrow\left[\left(\frac{p}{i w_{\mathrm{th}}}\right)^{n_{0}}, \psi(s, \sigma ; S \delta), \chi(\ell 0), \phi^{*}(J \delta)\right]_{w} \quad \text { and } \quad r^{\ell} \rightarrow f_{\ell}\left(\frac{r}{r_{0}}\right)
$$

where the first factor has already been given in (6.10). The right-hand side of the equations above are given entirely in unitless quantities, and they give the non-relativistic limit shown in (6.28) and (6.31).

We have presented several additional decay amplitudes in Appendix C, which lie outside the scope of the prescription for constructing covariant amplitudes as given in Section 6. The main reason for not including these amplitudes in the main text of this paper is because they violate the rule that an amplitude with $\ell$ must have a dependence $r^{\ell}$. If for some dynamical reason such an amplitude is needed, then we must keep in mind that the extra factors $r$ result from the time-components of the wave functions for $s$ and $\sigma$ [see (3.2)]. And hence they are fundamentally different from the $r^{\ell}$ dependence, which after all comes from a need to write down singularity-free amplitudes as $r \rightarrow 0$. As a result the procedure of substituting $r^{\ell}$ by a BW factor does not extend to the extra $r$ factors which are given in Appendix C.

It is clear, in retrospect, that the result of an earlier paper[1] by one of us applies only to the situation in which one or both of the decay products are massless particles, e.g. photons. In the paper it has been shown that the functions involving the Lorentz factors can expressed 
succinctly in a closed form. Unfortunately, such a closed expression seems impractical to us, and so we resort to the calculations on a case-by-case basis. It is hoped that the illustrative examples given in Sections 7, 8 and 9 are sufficiently diverse to give the reader how he should go about calculating the Lorentz factors for his own case. We have, in addition, posted a general $\mathrm{C}++$ program which gives the helicity-coupling amplitudes for any integer values of $J, s$ and $\sigma$, on two websites http://cern.ch/suchung and http://cern.ch/friedric, We have checked that all the results presented in the main text agree with the $\mathrm{C}++$ program. The reader is free to download for his own use. Any comments and/or queries on the program should be addressed to JF.

\section{Appendix A: The Lorentz Group in Four-Momentum Space}

The homogeneous Lorentz transformations are defined through

$$
p^{\prime \mu}=\Lambda_{\nu}^{\mu} p^{\nu} \quad \text { and } \quad g_{\mu \nu} \Lambda_{\rho}^{\mu} \Lambda_{\tau}^{\nu}=g_{\rho \tau}
$$

Our Lorentz metric $g^{\mu \nu}=g_{\mu \nu}$ has signature $(+,-,-,-)$.

$$
\begin{aligned}
& p^{\mu}=\left(E, p^{1}, p^{2}, p^{3}\right)=\left(E, p_{x}, p_{y}, p_{z}\right) \\
& p_{\mu}=g_{\mu \nu} p^{\nu}=\left(E, p_{1}, p_{2}, p_{3}\right)=\left(E,-p_{x},-p_{y},-p_{z}\right)
\end{aligned}
$$

Let $w$ be the mass associated with $p$ and adopt a notation in which $p$ indicates both the four-momentum and the magnitude of the 3-momentum, i.e.

$$
E^{2}=w^{2}+p^{2}, \quad p^{2}=p_{x}^{2}+p_{y}^{2}+p_{z}^{2}
$$

For each Lorentz transformation $\Lambda$, there exists a 'generalized angular momentum' operator $J^{\mu \nu}$ given by

$$
\Lambda=\exp \left[-\frac{i}{2} \omega_{\mu \nu} J^{\mu \nu}\right]
$$

where $\omega_{\mu \nu}$ is an antisymmetric matrix whose elements correspond to the six independent parameters of the Lorentz group. $J^{\mu \nu}$ is an antisymmetric operator imbedded in the fourmomentum space. It can be shown[7] that

$$
\left(J^{\mu \nu}\right)_{\sigma}^{\rho}=i\left(g^{\mu \rho} \delta_{\sigma}^{\nu}-g^{\nu \rho} \delta_{\sigma}^{\mu}\right)
$$


We define the angular momentum and the boost operator for $n=1,2$ or 3 via

$$
J^{n}=\frac{1}{2} \varepsilon^{n j k} J^{j k}, \quad K^{n}=J^{0 n}=-J^{n 0}
$$

where $\varepsilon^{123}=+1$. Explicitly, they can be expressed as

$$
\begin{aligned}
\left(J^{n}\right)_{\sigma}^{\rho} & =0, \quad \text { except } \quad\left(J^{n}\right)_{k}^{j}=-i \varepsilon_{n j k} \\
\left(K^{n}\right)_{\sigma}^{\rho} & =i\left(\delta_{\rho 0} \delta_{n \sigma}+\delta_{\rho n} \delta_{0 \sigma}\right)
\end{aligned}
$$

and they satisfy the usual commutation relations

$$
\left[J^{i}, J^{j}\right]=i \varepsilon^{i j k} J^{k}, \quad\left[J^{i}, K^{j}\right]=i \varepsilon^{i j k} K^{k}, \quad\left[K^{i}, K^{j}\right]=-i \varepsilon^{i j k} J^{k}
$$

The spin-1 wave functions at rest are

$$
e^{\mu}(0)=\left(\begin{array}{l}
0 \\
0 \\
0 \\
1
\end{array}\right), \quad e^{\mu}( \pm 1)=\mp \frac{1}{\sqrt{2}}\left(\begin{array}{c}
0 \\
1 \\
\pm i \\
0
\end{array}\right)
$$

It can be shown that, with $J_{x}=J^{1}, J_{y}=J^{2}$ and $J_{z}=J^{3}$,

$$
\left\{\begin{array}{l}
J^{2} e(m)=j(j+1) e(m), \quad j=1, \quad m=-1,0,+1 \\
J_{z} e(m)=m e(m), \quad m=-1,0,+1 \\
J_{ \pm} e(0)=\sqrt{2} e( \pm 1), \quad J_{ \pm} e(\mp 1)=\sqrt{2} e(0), \quad J_{ \pm} e( \pm 1)=0
\end{array}\right.
$$

where, with $J_{x}=J^{1}, J_{y}=J^{2}$ and $J_{z}=J^{3}, J^{2}=J_{x}^{2}+J_{y}^{2}+J_{z}^{2}$ and $J_{ \pm}=J_{x} \pm i J_{y}$. Consider a boost along the $z$-axis which takes the rest-state wave functions $e(m)$ to $e(\boldsymbol{p}, m)$, where $\boldsymbol{p}$ has only one nonzero component, i.e. the $z$-component. Again, with the notation $K_{x}=K^{1}$, $K_{y}=K^{2}$ and $K_{z}=K^{3}$, we can write $B_{z}(p)=\exp \left[-i \alpha K_{z}\right]$ and find

$$
\left[B_{z}(p)\right]_{\sigma}^{\rho}=\left(\begin{array}{cccc}
\cosh \alpha & 0 & 0 & \sinh \alpha \\
0 & 1 & 0 & 0 \\
0 & 0 & 1 & 0 \\
\sinh \alpha & 0 & 0 & \cosh \alpha
\end{array}\right),\left[B_{z}^{-1}(p)\right]_{\sigma}^{\rho}=\left(\begin{array}{cccc}
\cosh \alpha & 0 & 0 & -\sinh \alpha \\
0 & 1 & 0 & 0 \\
0 & 0 & 1 & 0 \\
-\sinh \alpha & 0 & 0 & \cosh \alpha
\end{array}\right)
$$


where $\cosh \alpha=E / w$ and $\sinh \alpha=p / w$. We see that

$$
e(\boldsymbol{p}, m)=B_{z}(p) e(m), \quad e^{\mu}(\boldsymbol{p}, 0)=\left(\begin{array}{l}
\eta \\
0 \\
0 \\
\gamma
\end{array}\right), \quad e^{\mu}(\boldsymbol{p}, \pm 1)=\mp \frac{1}{\sqrt{2}}\left(\begin{array}{c}
0 \\
1 \\
\pm i \\
0
\end{array}\right)
$$

where $\eta=p / w=\sinh \alpha=\gamma \beta$ and $\gamma=E / w=\cosh \alpha$ is the so-called Lorentz factor. The boosted wave functions $e(\boldsymbol{p}, m)$ satisfy the transversality condition

$$
p_{\mu} e^{\mu}(\boldsymbol{p}, m)=0
$$

The relativistic spin $W^{\mu}(p)$, operating on the states with an eigenvalue of $p^{\mu}$ is

$$
W^{\mu}(p)=\frac{1}{2} \varepsilon^{\mu \alpha \beta \gamma} p_{\alpha} J_{\beta \gamma}=\frac{1}{2} \varepsilon^{\mu \alpha \beta \gamma} J_{\alpha \beta} p_{\gamma}
$$

where we use the definition $\varepsilon_{0123}=+1$ so that $\varepsilon^{0123}=-1$. Here $p_{\mu}$ is not an operator. We see that

$$
\begin{aligned}
& W^{0}(p)=\boldsymbol{J} \cdot \boldsymbol{p} \\
& \boldsymbol{W}(p)=E \boldsymbol{J}+\boldsymbol{K} \times \boldsymbol{p}
\end{aligned}
$$

It can be shown that

$$
-W_{\mu}(p) W^{\mu}(p)=j(j+1) w^{2}, \quad j=1
$$

appropriate for particles with spin 1. We are now ready to define the 'total intrinsic spin' operator $S^{n}(p)$ via

$$
w S^{n}(p)=B_{z}(p) W^{n}(p) B_{z}^{-1}(p)
$$

confining ourselves to boosts along the z-axis only. It is clear by the definition that the actions of $w S^{n}(p)$ on $e(\boldsymbol{p}, m)$ are exactly the same as those of $w J$ on the at-rest states $e(m)$. And they obey the Lie algebra of angular momentum

$$
\left[S^{i}(p), S^{j}(p)\right]=i \epsilon^{i j k} S^{k}(p)
$$

Once again define $S^{1}(p)=S_{x}(p), S^{2}(p)=S_{y}(p)$ and $S^{3}(p)=S_{z}(p)$. It can be shown that

$$
w S_{z}(p)=w J_{z}, \quad w S_{x}(p)=E J_{x}+p K_{y}, \quad w S_{y}(p)=E J_{y}-p K_{x}
$$


With

$$
w^{2} S^{2}(p)=w^{2}\left[S_{x}^{2}(p)+S_{y}^{2}(p)+S_{z}^{2}(p)\right]
$$

we find

$$
\begin{gathered}
w S_{x}(p)=i\left(\begin{array}{cccc}
0 & 0 & +p & 0 \\
0 & 0 & 0 & 0 \\
+p & 0 & 0 & -E \\
0 & 0 & +E & 0
\end{array}\right), w S_{y}(p)=i\left(\begin{array}{cccc}
0 & -p & 0 & 0 \\
-p & 0 & 0 & +E \\
0 & 0 & 0 & 0 \\
0 & -E & 0 & 0
\end{array}\right) \\
w S_{z}(p)=w\left(\begin{array}{cccc}
0 & 0 & 0 & 0 \\
0 & 0 & -i & 0 \\
0 & +i & 0 & 0 \\
0 & 0 & 0 & 0
\end{array}\right), \quad w^{2} S^{2}(p)=2\left(\begin{array}{cccc}
-p^{2} & 0 & 0 & E p \\
0 & w^{2} & 0 & 0 \\
0 & 0 & w^{2} & 0 \\
-E p & 0 & 0 & E^{2}
\end{array}\right)
\end{gathered}
$$

Taking the usual raising and lowering operators $S_{ \pm}(p)=S_{x}(p) \pm i S_{y}(p)$, one can show that the boosted spin-1 wave functions $e(\boldsymbol{p}, m)$ given by (A.12) constitute the standard representation for the angular momentum operator $\vec{S}(p)$ defined above. To be more specific, the $4 \times 4$ matrices derived from $(\underline{\mathrm{A} .21} a)$ and $(\underline{\mathrm{A} .21} \mathrm{~b})$ acting on the 4-dimensional eigenvectors $e(\boldsymbol{p}, m)$ satisfy the familiar relationships for the operators $\left\{J_{ \pm}, J_{z}\right.$ and $\left.J^{2}\right\}$ acting on $|j m\rangle$ with $j=1$ [ see (A.10) $]$.

Define an arbitrary rotation by the Euler angles $\{\alpha, \beta, \gamma\}$

$$
R^{S}(\alpha, \beta, \gamma)=\exp \left[-i \alpha S_{z}(p)\right] \exp \left[-i \beta S_{y}(p)\right] \exp \left[-i \gamma S_{z}(p)\right]
$$

We see that

$$
R^{S}(\alpha, \beta, \gamma) e^{\mu}(\boldsymbol{p}, m)=\sum_{m^{\prime}} e^{\mu}\left(\boldsymbol{p}, m^{\prime}\right) D_{m^{\prime} m}^{(1)}(\alpha, \beta, \gamma)
$$

Note that the vector $\boldsymbol{p}$, defined to be along the $z$-axis, remain invariant through the rotation. Consider now a two-body system $(1+2)$ in a state of total intrinsic spin $S$, where the twobody $\vec{S}(p)$ is defined through

$$
S_{a}(p)=S_{a}^{(1)}(p)+S_{a}^{(2)}(p), \quad a=\{x, y, z\}
$$


and the two-body wave function in a state of total intrinsic spin $S$ is

$$
e^{\mu \nu}(\boldsymbol{p}, S m)=\sum_{m_{1} m_{2}}\left(1 m_{1} 1 m_{2} \mid S m\right) e^{\mu}\left(\boldsymbol{p}_{\mathbf{1}}, m_{1}\right) e^{\nu}\left(\boldsymbol{p}_{\mathbf{2}}, m_{2}\right)
$$

where $S=0,1$ or 2 and $\boldsymbol{p}=\boldsymbol{p}_{\mathbf{1}}+\boldsymbol{p}_{\mathbf{2}}$. We find

$$
R^{S}(\alpha, \beta, \gamma) e^{\mu \nu}(\boldsymbol{p}, S m)=\sum_{m^{\prime}} e^{\mu \nu}\left(\boldsymbol{p}, S m^{\prime}\right) D_{m^{\prime} m}^{S}(\alpha, \beta, \gamma)
$$

Note again that $\boldsymbol{p}$ is not affected by the rotation. In contrast, the orbital angular momentum operator $\boldsymbol{L}=\boldsymbol{J}-\boldsymbol{S}$ act only on $\boldsymbol{p}$ and does not affect the spin part of the wave function.

We have thus succeeded in separating out the total intrinsic part from the orbital angular momentum part in a two-body system.

\section{Appendix B: Spherical Harmonics}

Consider the breakup momentum $\vec{r}$ in the $J \mathrm{RF}$, whose direction is given by $\Omega=(\theta, \phi)$. A rank- $\ell$ tensor formed out of a single vector $r_{\alpha}$ can be contracted with the rank- $\ell$ tensor $\phi(\ell m)$ of (4.1). The resulting scalar is a function of $\Omega$ with quantum numbers $\ell$ and $m$; so it must be proportional to the spherical harmonics $Y_{\ell}^{m}(\Omega)$ :

$$
\begin{aligned}
& r^{\ell} Y_{\ell}^{m}(\Omega)=\sqrt{\frac{2 \ell+1}{4 \pi}}\left(c_{\ell}^{-1}\right)\left\{r_{\alpha} r_{\beta} r_{\gamma} \ldots\right\} \\
& \times\left\{\tilde{g}^{\alpha \alpha^{\prime}}(w) \tilde{g}^{\beta \beta^{\prime}}(w) \tilde{g}^{\gamma \gamma^{\prime}}(w) \ldots\right\} \\
& \times\left\{\phi_{\alpha^{\prime} \beta^{\prime} \gamma^{\prime} \ldots}(\ell m)\right\}
\end{aligned}
$$

where $c_{\ell}$ is that given by (4.10). (We show at the end of this appendix that the proportionality constant is indeed given by $\left(c_{\ell}\right)^{-1}$.) Define, in the $J \mathrm{RF}$,

$$
\left\{\begin{array}{l}
r \tau( \pm)=\vec{r} \cdot \vec{\phi}( \pm)=\mp \frac{r}{\sqrt{2}} \mathrm{e}^{ \pm i \phi} \sin \theta \\
r \tau(0)=\vec{r} \cdot \vec{\phi}(0)=r \cos \theta
\end{array}\right.
$$

so that

$$
Y_{\ell}^{m}(\Omega)=\sqrt{\frac{2 \ell+1}{4 \pi}}\left[\frac{(2 \ell) !}{2^{\ell}(\ell !)^{2}}\right]^{1 / 2} \phi\left\{\ell m ; \phi_{\alpha}(\nu) \rightarrow \tau(\nu)\right\}, \quad \nu=-1,0,+1
$$

Here $\phi(\ell m)$ is a not a tensor but a scalar in which each of the arguments is replaced by a scalar $\tau(m)$ (not a vector) given in (B.2). It is helpful to illustrate this with an example. 
The spherical harmonics for $\ell=1$ are readily obtained from (B.2). For $\ell=0$ and $\ell=2$, the general wave functions of (4.1), or more specifically the rank-2 tensor for spin-two wave functions given in (4.4), can be combined with (B.3), to find

$$
\left\{\begin{aligned}
Y_{0}^{0}(\Omega) & =\sqrt{\frac{1}{4 \pi}}, \quad Y_{1}^{1}(\Omega)=\sqrt{\frac{3}{4 \pi}} \tau(+)=-\sqrt{\frac{3}{8 \pi}} \mathrm{e}^{i \phi} \sin \theta \\
Y_{0}^{1}(\Omega) & =\sqrt{\frac{3}{4 \pi}} \tau(0)=\sqrt{\frac{3}{4 \pi}} \cos \theta \\
Y_{2}^{2}(\Omega) & =\sqrt{\frac{5}{4 \pi}} \sqrt{\frac{3}{2}} \tau(+) \tau(+)=\frac{1}{4} \sqrt{\frac{15}{2 \pi}} \mathrm{e}^{2 i \phi} \sin ^{2} \theta \\
Y_{2}^{1}(\Omega) & =\sqrt{\frac{5}{4 \pi}} \sqrt{\frac{3}{2}} \frac{1}{\sqrt{2}}[\tau(+) \tau(0)+\tau(0) \tau(+)]=-\sqrt{\frac{15}{8 \pi}} \mathrm{e}^{i \phi} \sin \theta \cos \theta \\
Y_{2}^{0}(\Omega) & =\sqrt{\frac{5}{4 \pi}} \sqrt{\frac{3}{2}} \frac{1}{\sqrt{6}}[\tau(+) \tau(-)+\tau(-) \tau(+)+2 \tau(0) \tau(0)] \\
& =\sqrt{\frac{5}{4 \pi}}\left(\frac{3}{2} \cos ^{2} \theta-\frac{1}{2}\right)
\end{aligned}\right.
$$

One can show that the formula holds for other values of $\ell$.

Define two real functions of $\theta$ by dropping the $\phi$ dependence in (B.2)

$$
\tau_{0}( \pm)=\mp \frac{1}{\sqrt{2}} \sin \theta, \quad \tau_{0}(0)=\cos \theta
$$

Noting that

we obtain, from (B.3),

$$
d_{m 0}^{\ell}(\theta)=\sqrt{\frac{4 \pi}{2 \ell+1}} Y_{\ell}^{m}(\Omega) \mathrm{e}^{-i m \phi}
$$

$$
d_{m 0}^{\ell}(\theta)=\left[\frac{(2 \ell) !}{2^{\ell}(\ell !)^{2}}\right]^{1 / 2} \phi\left\{\ell m ; \phi_{\alpha}(\nu) \rightarrow \tau_{0}(\nu)\right\}, \quad \nu=-1,0,+1
$$

For example, we find that $d_{m 0}^{\ell}(\theta)$ 's for $\ell=3$ are, from (4.5),

$$
\left\{\begin{aligned}
d_{30}^{3}(\theta) & =\sqrt{\frac{5}{2}} \tau_{0}(+) \tau_{0}(+) \tau_{0}(+)=-\frac{\sqrt{5}}{4} \sin ^{3} \theta \\
d_{20}^{3}(\theta) & =\sqrt{\frac{15}{2}} \tau_{0}(+) \tau_{0}(+) \tau_{0}(0)=\frac{1}{2} \sqrt{\frac{15}{2}} \sin ^{2} \theta \cos \theta \\
d_{10}^{3}(\theta) & =\sqrt{\frac{3}{2}}\left[\tau_{0}(+) \tau_{0}(+) \tau_{0}(-)+2 \tau_{0}(+) \tau_{0}(0) \tau_{0}(0)\right] \\
& =-\sqrt{3} \sin \theta\left(\frac{5}{4} \cos ^{2} \theta-\frac{1}{4}\right) \\
d_{00}^{3}(\theta) & =3 \tau_{0}(+) \tau_{0}(-) \tau_{0}(0)+\tau_{0}(0) \tau_{0}(0) \tau_{0}(0) \\
& =\left(\frac{5}{2} \cos ^{2} \theta-\frac{3}{2}\right) \cos \theta
\end{aligned}\right.
$$


In order to show that the proportionality constant is indeed $\left(c_{\ell}\right)^{-1}$, consider two special cases. First, set $\theta=0$. From the definition of the $d$-functions,

$$
d_{m 0}^{\ell}(\theta)=\left\langle\ell m\left|R_{y}(\theta)\right| \ell 0\right\rangle
$$

where $R_{y}(\theta)$ is a rotation by $\theta$ around the $y$-axis, we must have $d_{m 0}^{\ell}(0)=1$ for $m=0$ and $d_{m 0}^{\ell}(0)=0$ for $m \neq 0$. Since $\tau_{0}( \pm)=0$ and $\tau_{0}(0)=1$ for $\theta=0$, we find

$$
\phi\left\{\ell 0 ; \phi_{\alpha}(\nu) \rightarrow \tau(\nu)\right\}_{\theta=0}=\left[\frac{2^{\ell}(\ell !)^{2}}{(2 \ell) !}\right]^{1 / 2}
$$

We see that (B.7) is satisfied. Consider next the case of $\theta=\pi / 2$ and $m=\ell$. For the purpose, we write down the $d_{m 0}(\theta)$-functions as given by Rose[6],

$$
d_{m 0}^{\ell}(\theta)=(-)^{m}(\ell !)[(\ell+m) !(\ell-m) !]^{1 / 2} \sum_{k=k_{1}}^{k_{2}} \frac{(-)^{k} \cos ^{2 \ell-n}(\theta / 2) \sin ^{n}(\theta / 2)}{(\ell-m-k) !(\ell-k) !(m+k) ! k !}
$$

where $n=m+2 k$ and $k$ is a non-negative integer ranging between $k_{1}$ and $k_{2}$ given by

$$
k_{1}=0, \quad k_{2}=\min \{\ell-m, \ell\}
$$

Since $m=\ell$, we see that $k_{1}=k_{2}=0$. So the sum in (B.11) reduces to just one term with $k=0$. We find, noting that $\sin (\theta / 2)=\cos (\theta / 2)=1 / \sqrt{2}$,

$$
d_{\ell 0}^{\ell}(\pi / 2)=(-)^{\ell}\left[\frac{(2 \ell) !}{2^{2 \ell}(\ell !)^{2}}\right]^{1 / 2}
$$

On the other hand, we obtain, noting that $\sin (\pi / 2)=1$,

$$
\phi\left\{\ell \ell ; \phi_{\alpha}(\nu) \rightarrow \tau(\nu)\right\}_{\theta=\pi / 2}=\left.\underbrace{\tau_{0}(+) \tau_{0}(+) \cdots}_{\ell}\right|_{\theta=\pi / 2}=(-)^{\ell}\left(\frac{1}{\sqrt{2}}\right)^{\ell}
$$

Here again we see that the proportionality constant given in (B.7) is satisfied.

We have worked out the spherical harmonics for $\ell=0,1$ and 2 ; and the $d$-functions for $\ell=3$, which have been checked with standard references on such mathematical functions. We have, in addition, shown that (B.7) is true for two special cases of arbitrary integer $\ell$. We have thus shown that (B.7) must be true in general, with the proportionality constant being independent of both $\theta$ and $m$. 


\section{Appendix C: Additional Decay Amplitudes}

The purpose of this appendix is explore a few decay amplitudes $A$ not included in the main text. They are exluded from our list of allowed amplitudes, because they induce in $A$ an additional dependence on $r$, which are exluded according to the $r^{\ell}$ rule as given in the prescriptions following (6.9). Consider the decay $J \rightarrow s+\sigma$, where $J=1,2, s=1,2$ and $\sigma=0$ or 1 . We consider $\ell=1$ only. In the vector notation, we have $\vec{J}=\vec{S}+\vec{\ell}$ and $\vec{S}=\vec{s}+\vec{\sigma}$.

The first case we wish to work out is for $\sigma=0$, i.e.

$$
1^{-} \rightarrow 1^{-}+0^{-}
$$

The decay amplitude we want to consider here is, with $J=1, S=s=1$ and $\ell=1$,

$$
A_{\ell s}^{J}(s \delta)=r_{\alpha} \omega^{\alpha}(s \delta) \chi^{\beta}(\ell 0) \phi_{\beta}^{*}(J \delta)
$$

In the JRF, the decay amplitudes are

$$
\begin{aligned}
A_{\ell s}^{J}(s \delta) & =0, \quad \delta= \pm 1 \\
& =\left(\frac{W}{2 m}\right) r, \quad \delta=0
\end{aligned}
$$

We do not include this decay amplitude because it apparently violates the parity conservation, since the full decay amplitude $F^{J}$ should be proportional to $r$ for $\ell=1$ and not $r^{2}$. The second case we examine for $\sigma=0$ is

$$
1^{+} \rightarrow 2^{+}+0^{-}
$$

with its decay amplitude given by

$$
A_{\ell s}^{J}(s \delta)=p_{\alpha} p_{\beta} \omega^{\alpha \beta}(s \delta) \chi^{\gamma}(\ell 0) \phi_{\gamma}^{*}(J \delta)
$$

In the $J R F$, the decay amplitudes are

$$
\begin{aligned}
A_{\ell s}^{J}(s \delta) & =0, \quad \delta= \pm 1 \\
& =\frac{1}{2 \sqrt{6}}\left(\frac{W^{2}}{m \mu}\right) r^{2}, \quad \delta=0
\end{aligned}
$$

This does not violate parity but the full decay amplitude $F^{J}$ is now proportional to $r^{3}$ for an $\ell=1$ decay mode. For this reason, we do not allow such a decay amplitude. 
We now go over to the case $\sigma=1$ and consider the decay

$$
1^{+} \rightarrow 1^{+}+1^{-}
$$

We wish to work out the following invariant amplitude

$$
A_{\ell S}^{J}(S \delta)=\epsilon_{\mu \alpha \beta \gamma} \psi^{\mu \alpha}(S \delta) \chi^{\beta}(\ell 0) \phi^{* \gamma}(J \delta)
$$

where $\epsilon_{0123}=+1$ (so that $\left.\epsilon^{0123}=-1\right)$ and

$$
\psi^{\mu \alpha}(S \delta)=\sum_{m_{a} m_{b}}\left(1 m_{a} 1 m_{b} \mid S \delta\right) \omega^{\mu}\left(s m_{a}\right) \varepsilon^{\alpha}\left(\sigma m_{b}\right), \quad \delta=m_{a}+m_{b}
$$

Introducing a shorthand notation

$$
[a b c]=\epsilon_{i j k} a^{i} b^{j} c^{k}=(\vec{a} \cdot \vec{b} \times \vec{c})=(\vec{a} \times \vec{b} \cdot \vec{c})
$$

so that

$$
[a b c d] \equiv \epsilon_{\mu \alpha \beta \gamma} a^{\mu} b^{\alpha} c^{\gamma} d^{\delta}=a^{0}[b c d]-b^{0}[a c d]+c^{0}[a b d]-d^{0}[a b c]
$$

and we can write, in the $J R F$,

$$
\begin{aligned}
A_{\ell S}^{J}(S \delta)= & \sum_{m_{a} m_{b}}\left(1 m_{a} 1 m_{b} \mid S \delta\right) \epsilon_{\mu \alpha \beta \gamma} \omega^{\mu}\left(s m_{a}\right) \varepsilon^{\alpha}\left(\sigma m_{b}\right) \chi^{\beta}(\ell 0) \phi^{* \gamma}(J \delta) \\
= & \sum_{m_{a} m_{b}}\left(1 m_{a} 1 m_{b} \mid S \delta\right) \\
\times & \left\{\omega^{0}\left(s m_{a}\right)\left[\varepsilon\left(\sigma m_{b}\right) \chi(\ell 0) \phi^{*}(J \delta)\right]\right. \\
- & {\left.\left[\omega\left(s m_{a}\right) \chi(\ell 0) \phi^{*}(J \delta)\right] \varepsilon^{0}\left(\sigma m_{b}\right)\right\} }
\end{aligned}
$$

since $\chi^{0}=0$ and $\phi^{0}=0$ in the $J$ RF. 
This leads to, dropping $J, s$ and $\sigma$ for compact notation,

$$
\begin{aligned}
A_{\ell S}^{J}(S 0) & =0 \\
A_{\ell S}^{J}(S+) & =(1011 \mid S 1) \omega^{0}(0)\left[\varepsilon(+) \chi(0) \phi^{*}(+)\right]-(1110 \mid S 1)\left[\omega(+) \chi(0) \phi^{*}(+)\right] \varepsilon^{0}(0) \\
& =(i)\left[(1011 \mid S 1) \omega^{0}(0)-(1110 \mid S 1) \varepsilon^{0}(0)\right] \\
& =(i)\left[(1011 \mid S 1) \gamma_{s} \beta_{s}+(1110 \mid S 1) \gamma_{\sigma} \beta_{\sigma}\right] \\
& =0, \quad S=0 \\
& =\frac{i}{\sqrt{2}}\left(-\gamma_{s} \beta_{s}+\gamma_{\sigma} \beta_{\sigma}\right)=\frac{i r}{2 \sqrt{2}}\left(-\frac{1}{m}+\frac{1}{\mu}\right), \quad S=1 \\
& =\frac{i}{\sqrt{2}}\left(\gamma_{s} \beta_{s}+\gamma_{\sigma} \beta_{\sigma}\right)=\frac{i r}{2 \sqrt{2}}\left(\frac{1}{m}+\frac{1}{\mu}\right), \quad S=2
\end{aligned}
$$

These amplitudes lead to a new term to the amplitudes given in (6.17). They are

$$
\bar{g}_{11}^{(1)} r \quad \text { and } \quad \bar{g}_{12}^{(1)} r
$$

where $\bar{g}$ 's are new parameters in the problem. Note that the decay-coupling amplitudes $F^{J}$ for $\ell=1$ are proportional to $r^{2}$, and hence they violate the rule that we have adopted in this paper, namely that the $F^{J}$ for a given given $\ell=2$ are always proportional to $r^{\ell}$.

We now consider another additional decay amplitude which have not been included in the this paper. It comes from allowing more than one factor of $p$ to be used in constructing decay amplitudes. Consider

$$
A_{\ell S}^{J}(S \delta)=p_{\mu} p_{\alpha} \psi^{\mu \alpha}(S \delta) \chi^{\beta}(\ell 0) \phi_{\beta}^{*}(J \delta)
$$

In the $J R F$, this leads to

$$
\begin{aligned}
A_{\ell S}^{J}(S+) & =0 \\
A_{\ell S}^{J}(S 0) & =(1010 \mid S 0) w^{2}\left(\gamma_{s} \beta_{s}\right)\left(-\gamma_{\sigma} \beta_{\sigma}\right)=-(1010 \mid S 0)\left(\frac{w^{2}}{4 m \mu}\right) r^{2} \\
& =\frac{1}{\sqrt{3}}\left(\frac{w^{2}}{4 m \mu}\right) r^{2}, \quad S=0 \\
& =0, \quad S=1 \\
& =-\sqrt{\frac{2}{3}}\left(\frac{w^{2}}{4 m \mu}\right) r^{2}, \quad S=2
\end{aligned}
$$


These amplitudes lead to a new term to the amplitudes given in (6.17). They are

$$
\bar{g}_{10}^{(1)}\left(\frac{w^{2}}{m \mu}\right) r^{3} \quad \text { and } \quad \hat{g}_{12}^{(1)}\left(\frac{w^{2}}{m \mu}\right) r^{3}
$$

where $\bar{g}$ and $\hat{g}$ are new parameters in the problem. Again these decay amplitudes have not been included in the Section 7 of this paper, because the decay-coupling amplitudes $F^{J}$ for $\ell=1$ are proportional to $r^{3}$.

We consider the additional decay amplitudes considered in this section to be 'anomalous.' They are highly relativistic, as they vanish faster than those treated in the Section 7 as $r \rightarrow 0$. Conversely, they may dominate the decay amplitudes as $w \rightarrow \infty$ and therefore must be included in any general treatment of the problem.

There exist two additional anomalous amplitudes for the decay

$$
2^{-} \rightarrow 1^{+}+1^{-}, \quad \ell=2
$$

One of them is, evidently,

$$
A_{\ell S}^{J}(S \delta)=\epsilon_{\mu \alpha \beta \gamma} \psi^{\mu \alpha}(S \delta) \chi^{\beta \rho}(\ell 0) \tilde{g}_{\rho \tau}(w) \phi^{* \tau \gamma}(J \delta)
$$

It takes on the form, in the $J R F$,

$$
\begin{aligned}
A_{\ell S}^{J}(S \delta)=\sum_{m_{a} m_{b}}\left(1 m_{a} 1 m_{b} \mid S \delta\right) \epsilon_{\mu \alpha \beta \gamma} \omega^{\mu}\left(s m_{a}\right) \varepsilon^{\alpha}\left(\sigma m_{b}\right) \chi^{\beta \rho}(\ell 0) \tilde{g}_{\rho \tau}(w) \phi^{* \tau \gamma}(J \delta) \\
=\sum_{m_{a} m_{b}}\left(1 m_{a} 1 m_{b} \mid S \delta\right) \\
\times\left\{\omega^{0}\left(s m_{a}\right)\left[\varepsilon\left(\sigma m_{b}\right) \chi(\ell 0) \cdot \phi^{*}(J \delta)\right]\right. \\
\left.-\left[\omega\left(s m_{a}\right) \chi(\ell 0) \cdot \phi^{*}(J \delta)\right] \varepsilon^{0}\left(\sigma m_{b}\right)\right\}
\end{aligned}
$$


so that

$$
\begin{aligned}
& A_{\ell S}^{J}(S 2)= 0 \\
& \begin{aligned}
& A_{\ell S}^{J}(S 1)= \frac{1}{3 \sqrt{2}} \sum_{m_{a} m_{b}}\left(1 m_{a} 1 m_{b} \mid S 1\right) \\
& \times\left\{\omega^{0}\left(m_{a}\right)\left[\varepsilon\left(m_{b}\right)\left(\chi(-) \phi^{*}(0)+2 \chi(0) \phi^{*}(+)\right)\right]\right. \\
&\left.-\left[\omega\left(m_{a}\right)\left(\chi(-) \phi^{*}(0)+2 \chi(0) \phi^{*}(+)\right)\right] \varepsilon^{0}\left(m_{b}\right)\right\} \\
&= \frac{1}{6}(-)^{S}\left\{\omega^{0}(0)\left[\varepsilon(+)\left(\chi(-) \phi^{*}(0)+2 \chi(0) \phi^{*}(+)\right)\right]\right\} \\
&-\frac{1}{6}\left\{\left[\omega(+)\left(\chi(-) \phi^{*}(0)+2 \chi(0) \phi^{*}(+)\right)\right] \varepsilon^{0}(0)\right\} \\
&=\left(\frac{i}{3}\right)\left[(-)^{S} \gamma_{s} \beta_{s}+\gamma_{\sigma} \beta_{\sigma}\right]=\left(\frac{i r}{6}\right)\left[\frac{(-)^{S}}{m}+\frac{1}{\mu}\right] \\
& A_{\ell S}^{J}(S 0)=0
\end{aligned}
\end{aligned}
$$

So the decay-coupling amplitudes $F^{J}$ for $\ell=2$ have a term proportional to $r^{3}$. The second anomalous amplitude is

$$
A_{\ell S}^{J}(S \delta)=p_{\mu} p_{\alpha} \psi^{\mu \alpha}(S \delta) \chi(\ell 0)^{\nu \beta} \phi_{\nu \beta}^{*}(J \delta)
$$

which leads to, in the JRF,

$$
\begin{aligned}
A_{\ell S}^{J}(S 2) & =A_{\ell S}^{J}(S 1)=0 \\
A_{\ell S}^{J}(S 0) & =-\sqrt{\frac{2}{3}}(1010 \mid S 0)\left(\frac{w^{2}}{4 m \mu}\right) r^{2} \\
& =\frac{\sqrt{2}}{3}\left(\frac{w^{2}}{4 m \mu}\right) r^{2}, \quad S=0 \\
& =0, \quad S=1 \\
& =-\frac{2}{3}\left(\frac{w^{2}}{4 m \mu}\right) r^{2}, \quad S=2
\end{aligned}
$$

Here the decay-coupling amplitudes $F^{J}$ for $\ell=2$ have a term proportional to $r^{4}$. 


\section{Acknowledgments}

S. U. Chung is indebted to the German Ministry of Science and Technology (BMBF) and the DFG for providing him with an appointment of Visiting Professorship for the years 2003 through 2005 and again for the years 2007 through 2009. This work started in 2003 during his visits to Bonn and Munich under the sponsorship. He acknowledges with gratitude the financial support in 2007 as scientific consultant to the Cluster of Excellence for Fundamental Physics in Munich, Germany.

He is also indebted to Larry Trueman/BNL and Ron Longacre/BNL for their numerous helpful comments during the final stages of this paper.

Finally, both of us acknowledge with pleasure the support and encouragement from Professor Stephan Paul, Technical University Munich.

\section{References}

[1] S. U. Chung, Phys. Rev. D57, 431(1998).

[2] S. U. Chung, Phys. Rev. D48, 1225(1993); Phys. Rev. D56, 4419(1997).

[3] S. U. Chung, 'Spin Formalisms,' CERN Yellow Report 71-8 (1971).

[4] A. McKerrell, Nuovo Cimento 34, 1289 (1964).

[5] A. J. Macfarlane, J. Math. Phys. 4, 490 (1963).

[6] M.E. Rose, Elementary theory of angular momentum (John Wiley \& Sons, Inc., New York, 1957).

[7] M. Maggiore, 'A Modern Introduction to Quantum Field Theory, (Oxford University Press, Oxford, 2005), Chapter 2.

[8] M. Jacob and G. C. Wick, Ann. Phys. (N.Y.) 7, 404 (1959).

[9] W. Rarita and J. Schwinger, Phys. Rev. 60, 61 (1941). 
[10] R. E. Behrends and C. Fronsdal, Phys. Rev. 106, 345 (1957);

C. Fronsdal, Nuovo Cimento Suppl. $\underline{9}, 416$ (1958).

[11] C. Zemach, Phys. Rev. 140, B97 (1965); ibid. 140, B109 (1965)

[12] B. W. Lee, C. Quigg, and H. B. Thacker, Phys. Rev. D 16, 1519 (1977); M. S. Chanowitz and M. K. Gaillard, Nucl. Phys. B261, 379 (1985); G. J. Gounaris, R. Kögler and H. Neufeld, Phys. Rev. D 34, 3257 (1986); W. Marciano and S. Willenbrock, ibid. 37, 2509 (1988).

[13] F. von Hippel and C. Quigg, Phys. Rev. $\underline{5}, 624$ (1972). 